TRANSACTIONS OF THE

AMERICAN MATHEMATICAL SOCIETY

Volume 348, Number 7, July 1996

\title{
REPRESENTATIONS OF MONOIDS ARISING FROM FINITE GROUPS OF LIE TYPE
}

\author{
A. SALWA
}

\begin{abstract}
A class of finite monoids $M$ constructed from a group $G$ of Lie type is considered. We describe the irreducible complex representations and prove the complete reducibility of the representations of $M$. The sandwich matrix of $M$ is decomposed into a product of matrices corresponding to maximal parabolic subgroups of $G$.
\end{abstract}

\section{INTRODUCTION}

Monoids of Lie type were introduced in [7] as finite analogues of linear algebraic monoids. They were used to solve the long-standing problem on the semisimplicity of the complex algebra of the full matrix monoid $M_{n}\left(F_{q}\right)$ over a finite field $F_{q}$, [6]. Their connections with the representation theory and combinatorics of finite groups of Lie type, via so called sandwich matrices, then became apparent and were extensively studied in $[9,10,12]$. In particular, this approach has recently led to a new explicit description of the Steinberg representation, [11] .

In this paper we consider three $\mathcal{J}$-class monoids $M=M\left(G, P, P^{-}, L\right)=G \sqcup$ $J \sqcup\{0\}$ where $G$ is the group of units and any two idempotents in $J$ are conjugate. Moreover we assume that $G$ is a finite group with a split $B N$-pair satisfying some commutator relations. $P, P^{-}$are parabolic subgroups of $G$ with a common Levi factor $L$. In the case where $P$ and $P^{-}$are opposite, these monoids are called universal three $\mathcal{J}$-class monoids of Lie type and give the local structure of any monoid of Lie type, $[7,8]$. We prove that the complex semigroup algebra $\mathbf{C}_{0}[M]$ is semisimple in the general case. This is done by proving that certain $\mathbf{C}[M]$ modules are irreducible, which turns out to be equivalent to showing that some homomorphisms of $\mathbf{C}[G]$-modules are in fact isomorphisms. These homomorphisms were used to construct the standard bases of Hecke algebras in the cuspidal case, [4]. So the semisimplicity problem for $\mathbf{C}_{0}[M]$ is formulated in terms of group representation theory. Moreover all irreducible representations of $M$ are described explicitly. In the last section certain decomposition of the sandwich matrix of $M$ is obtained. This reduces the problem of finding the inverse of this matrix to the case where $P$ and $P^{-}$are maximal and opposite. It is worth mentioning that this case is crucial for the motivating example $M=M_{n}\left(F_{q}\right)$ which was considered in $[5,6]$. In particular this shows that considering our class of monoids, wider than monoids of Lie type, is natural since it allows an induction. Our techniques are built on

Received by the editors February 3, 1995 and, in revised form, September 18, 1995.

1991 Mathematics Subject Classification. Primary 20M30, 20M25.

Supported by KBN research grant 2 P301 05106. 
representation theory of finite groups of Lie type. The monograph of Carter, [1], will be the standard reference.

\section{IRREDUCIBLE REPRESENTATIONS}

We briefly review some basics of semigroup theory, see [2] for details. Let $M$ be a monoid (i.e. has an identity element). $\mathcal{J}$ will denote one of Green's equivalence relations on $M: a \mathcal{J} b$ if $M a M=M b M$. If $J$ is a $\mathcal{J}$-class of $M$, then define $J^{0}=J \cup\{0\}$ with

$$
a \cdot b= \begin{cases}a b & \text { if } a b \in J \\ 0 & \text { otherwise. }\end{cases}
$$

If $M$ is finite then $J^{0}$ is either a null semigroup or else a completely 0-simple semigroup. Any completely 0 -simple semigroup has a Rees representation $\mathcal{M}\left(H, I_{1}, I_{2}, \mathcal{P}\right)$ where $H$ is a maximal subgroup and $\mathcal{P}$ is an $I_{2} \times I_{1}$ sandwich matrix with entries in $H \cup\{0\}$.

Let $G$ be a finite group with subgroups $P, P^{-}$and homomorphisms $\delta: P \rightarrow$ $L, \delta^{-}: P^{-} \rightarrow L$ onto a finite group $L$ such that $\left.\delta\right|_{P \cap P^{-}}=\left.\delta^{-}\right|_{P \cap P^{-}}$. We can then construct a finite three $\mathcal{J}$-class monoid $M=M\left(G, P, P^{-}, L\right)=G \sqcup J \sqcup\{0\}$. Here $J=(G \times L \times G) / \equiv$ with $s_{1} \equiv s_{2}$ defined for $s_{1}=\left(x_{1}, l_{1}, y_{1}\right), s_{2}=\left(x_{2}, l_{2}, y_{2}\right) \in$ $G \times L \times G$ by the conditions $x_{2}^{-1} x_{1} \in P, y_{2} y_{1}^{-1} \in P^{-}$and $\delta\left(x_{2}^{-1} x_{1}\right) l_{1}=l_{2} \delta^{-}\left(y_{2} y_{1}^{-1}\right)$. For $s=(x, l, y), \bar{s}=(\bar{x}, \bar{l}, \bar{y}) \in J, g \in G$ the multiplication rule is given by:

$$
\begin{gathered}
s \bar{s}= \begin{cases}\left(x, l \delta^{-}(p) \delta(q) \bar{l}, \bar{y}\right) & \text { if } y \bar{x}=p q, p \in P^{-}, q \in P, \\
0 & \text { if } y \bar{x} \notin P^{-} P\end{cases} \\
s g=(x, l, y g), \quad g s=(g x, l, y) .
\end{gathered}
$$

Monoids of this type were defined and investigated by Putcha, cf. [8]. Consider the coset decompositions $G=\bigsqcup_{j=1}^{n} P^{-} a_{j}=\bigsqcup_{i=1}^{m} b_{i} P, a_{1}=1, b_{1}=1$. Every element of $J$ is uniquely expressible in the form $\left(b_{i}, l, a_{j}\right)$ where $b_{i}, a_{j}$ are as above and $l \in L$. If $e$ denotes the idempotent $(1,1,1)$, then $J=G e G$. We say that $s, \bar{s} \in M$ are conjugate if $g s g^{-1}=\bar{s}$ for some $g \in G$. It is easy to see that any two idempotents in $J$ are conjugate. In some sense the converse is also true, cf. [8]. $J$ is a $\mathcal{J}$ class of $M$ and $J^{0}$ is a completely 0 -simple semigroup. $J^{0}$ has a Rees presentation $\mathcal{M}\left(L, I_{1}, I_{2}, \mathcal{P}\right)$ where $I_{1}=\left\{b_{1}, b_{2}, \ldots, b_{m}\right\}, I_{2}=\left\{a_{1}, a_{2}, \ldots, a_{n}\right\}$ and the sandwich matrix $\mathcal{P}=\left(p_{j, i}\right)$ is defined by

$$
p_{j, i}= \begin{cases}\delta^{-}(p) \delta(q) & \text { if } a_{j} b_{i}=p q, p \in P^{-}, q \in P, \\ 0 & \text { if } a_{j} b_{i} \notin P^{-} P .\end{cases}
$$

Note that $\mathcal{P}$ depends on the choice of coset representatives.

Let $K$ be a field. By a representation of $M$ we will mean a homomorphism $\rho: M \rightarrow M_{n}(K)$ such that $\rho(1)=1$ and $\rho(0)=0$. Let $K_{0}[M]=K[M] / K 0_{M}$ denote the contracted semigroup algebra of $M$. Then the representations of $M$ are in a natural one-to-one correspondence with left $K_{0}[M]$-modules.

Let $X$ be an irreducible $K[L]$-module. Let $V$ be the linear space over $K$ with basis $b_{1}, b_{2}, \ldots, b_{m}$. Define $\bar{X}=V \otimes X$, where the tensor product is over $K$. We may give $\bar{X}$ the structure of a $K[M]$-module as follows :

$$
s\left(b_{i} \otimes x\right)=b_{s(i)} \otimes \gamma(s, i) x \text { for } s \in M, x \in X
$$

where $\gamma(s, i)$, and $s(i)$ if $\gamma(s, i) \neq 0$, are defined by

$$
s\left(b_{i}, 1,1\right)=\left(b_{s(i)}, \gamma(s, i), 1\right) \text { with } \gamma(s, i) \in L \cup\{0\}
$$


(every $(x, 0, y)$ will be identified with zero of $M$, so $s(i)$ need not be defined for $\gamma(s, i)=0)$. That the action is well-defined is obvious. We must show that

$$
s_{1}\left[s_{2}\left(b_{i} \otimes x\right)\right]=\left(s_{1} s_{2}\right)\left(b_{i} \otimes x\right) \text { for } s_{i} \in M, x \in X .
$$

First, note that $s_{2}\left(b_{i}, 1,1\right)=\left(b_{s_{2}(i)}, \gamma\left(s_{2}, i\right), 1\right)$. Thus, if $0 \neq \gamma\left(s_{2}, i\right)=\delta^{-}(q)$ for some $q \in P^{-}$, we have

$$
\begin{gathered}
\left(s_{1} s_{2}\right)\left(b_{i}, 1,1\right)=s_{1}\left(b_{s_{2}(i)}, \gamma\left(s_{2}, i\right), 1\right)=s_{1}\left(b_{s_{2}(i)}, \delta^{-}(q), 1\right) \\
=s_{1}\left(b_{s_{2}(i)}, 1,1\right) q=\left(b_{s_{1}\left(s_{2}(i)\right)}, \gamma\left(s_{1}, s_{2}(i)\right), 1\right) q=\left(b_{s_{1}\left(s_{2}(i)\right)}, \gamma\left(s_{1}, s_{2}(i)\right) \delta^{-}(q), 1\right) \\
=\left(b_{s_{1}\left(s_{2}(i)\right)}, \gamma\left(s_{1}, s_{2}(i)\right) \gamma\left(s_{2}, i\right), 1\right) .
\end{gathered}
$$

The equality

$$
\left(s_{1} s_{2}\right)\left(b_{i}, 1,1\right)=\left(b_{s_{1}\left(s_{2}(i)\right)}, \gamma\left(s_{1}, s_{2}(i)\right) \gamma\left(s_{2}, i\right), 1\right)
$$

also holds if $\gamma\left(s_{2}, i\right)=0$. Furthermore $\left(s_{1} s_{2}\right)\left(b_{i}, 1,1\right)=\left(b_{\left(s_{1} s_{2}\right)(i)}, \gamma\left(s_{1} s_{2}, i\right), 1\right)$. This implies that

$$
\begin{gathered}
s_{1}\left(s_{2}(i)\right)=\left(s_{1} s_{2}\right)(i) \text { if } \gamma\left(s_{1} s_{2}, i\right) \neq 0, \\
\gamma\left(s_{1}, s_{2}(i)\right) \gamma\left(s_{2}, i\right)=\gamma\left(s_{1} s_{2}, i\right) .
\end{gathered}
$$

Therefore (1) follows :

$$
\begin{aligned}
s_{1}\left[s_{2}\left(b_{i} \otimes x\right)\right] & =s_{1}\left(b_{s_{2}(i)} \otimes \gamma\left(s_{2}, i\right) x\right)=b_{s_{1}\left(s_{2}(i)\right)} \otimes \gamma\left(s_{1}, s_{2}(i)\right) \gamma\left(s_{2}, i\right) x \\
& =b_{\left(s_{1} s_{2}\right)(i)} \otimes \gamma\left(s_{1} s_{2}, i\right) x=\left(s_{1} s_{2}\right)\left(b_{i} \otimes x\right) .
\end{aligned}
$$

Note that $\bar{X}$ is related to Schutzenberger representations, [2, Section 3.5]. Our aim is to describe irreducible representations of $M$ explicitly in the case where $K_{0}[M]$ is semisimple. The general theory of representations of semigroups, [2, chapter 5], cannot be applied since this requires the exact form of the inverse of $\mathcal{P}$ over $K[L]$. $\mathcal{P}^{-1}$ is known in some special cases only, cf. [9].

With the above notation we can state our first result.

Theorem 2.1. Assume that $K$ is an algebraically closed field of characteristic zero and $\left|P^{-}\right| \geq|P|$. Then the monoid algebra $K_{0}[M]$ is semisimple if and only if all $K[M]$-modules $\bar{X}$ (for all irreducible $K[L]$-modules $X$ ) are irreducible. Moreover, in this case all irreducible $K[M]$-modules with e acting not as 0 are of this type.

Proof. Suppose first that all $K[M]$-modules $\bar{X}$ are irreducible. Let $W_{1}, W_{2}, \ldots, W_{r}$ $\left(V_{1}, V_{2}, \ldots, V_{s}\right.$ respectively) be representatives of isomorphism classes of irreducible modules over $K[G](K[L])$. Then $W_{1}, W_{2}, \ldots, W_{r}$ are also $K[M]$-modules with $K[J]$ acting as zero. We will prove that $K[M]$-modules $W_{1}, W_{2}, \ldots, W_{r}, \bar{V}_{1}$, $\bar{V}_{2}, \ldots, \bar{V}_{s}$ are pairwise nonisomorphic. It is sufficient to show that $\bar{V}_{i} \not \bar{V}_{j}$ for $i \neq j$. First, note that

$$
e\left(b_{i}, 1,1\right)= \begin{cases}\left(b_{1}, \delta^{-}\left(p^{-}\right) \delta(p), 1\right) & \text { if } b_{i}=p^{-} p \text { for some } p^{-} \in P^{-}, p \in P, \\ 0 & \text { otherwise. }\end{cases}
$$

This gives $e(1)=1$ if $\gamma(e, i) \neq 0$. Hence $e\left(b_{i} \otimes x\right)=b_{1} \otimes \gamma(e, i) x$ for $x \in V_{k}$ and then $e \bar{V}_{k} \subseteq b_{1} \otimes V_{k}$. Since $e\left(b_{1} \otimes x\right)=b_{1} \otimes x$ for $x \in V_{k}$, we have $e \bar{V}_{k}=b_{1} \otimes V_{k}$. Let us define $\varphi: e \bar{V}_{k}=b_{1} \otimes V_{k} \rightarrow V_{k}$ by $\varphi\left(b_{1} \otimes v\right)=v, v \in V_{k}$. Then $e \bar{V}_{k}$ is a $K[(1, L, 1)]=K[L]$-module and $\varphi$ is an isomorphism of $K[L]$-modules. If $K[M]$-modules $\bar{V}_{i}, \bar{V}_{j}$ are isomorphic, then $K[L]$-modules $e \bar{V}_{i}, e \bar{V}_{j}$ are isomorphic. Hence $K[L]$-modules $V_{i}, V_{j}$ are isomorphic and $i=j$. Since $K[G]$ is semisimple, 
$\sum_{i=1}^{r}\left(\operatorname{dim}_{K} W_{i}\right)^{2}=|G|$. We continue in this fashion, obtaining $\sum_{i=1}^{s}\left(\operatorname{dim}_{K} \bar{V}_{i}\right)^{2}=$ $\sum_{i=1}^{s}\left(|G / P| \operatorname{dim}_{K} V_{i}\right)^{2}=|G / P|^{2}|L|$ since $K[L]$ is semisimple. Hence

$$
\sum_{i=1}^{r}\left(\operatorname{dim}_{K} W_{i}\right)^{2}+\sum_{i=1}^{s}\left(\operatorname{dim}_{K} \bar{V}_{i}\right)^{2} \geq|G|+|G / P \| L|\left|G / P^{-}\right|=\operatorname{dim}_{K} K_{0}[M] .
$$

This proves that $K_{0}[M]$ is semisimple $\left(|P|=\left|P^{-}\right|\right.$in particular) and all irreducible $K[M]$-modules are among $W_{1}, W_{2}, \ldots, W_{r}, \bar{V}_{1}, \bar{V}_{2}, \ldots, \bar{V}_{s}$.

Conversely, assume that $K_{0}[M]$ is semisimple. Let $X$ be an irreducible $K[L]$ module. Define $\bar{X}_{0}=\{v \in \bar{X}:(G e G) v=0\}$. $\bar{X}_{0}$ is a $K[M]$-submodule of $\bar{X}$. Since $K_{0}[M]$ is semisimple there exists a $K[M]$-submodule $\bar{X}_{1}$ of $\bar{X}$ such that $\bar{X}_{0} \oplus \bar{X}_{1}=\bar{X}$. Then $e \bar{X}=e \bar{X}_{0}+e \bar{X}_{1}=e \bar{X}_{1} \subseteq \bar{X}_{1}$. Thus $b_{1} \otimes X \subseteq \bar{X}_{1}$. This gives $K[G]\left(b_{1} \otimes X\right) \subseteq \bar{X}_{1}$. Since also $K[G]\left(b_{1} \otimes x\right)=\bar{X}$, we have $\bar{X}_{1}=\bar{X}$ and so $\bar{X}_{0}=0$. Let $v \in \bar{X} \backslash\{0\}$. Then there exists $g \in G$ such that egv $\neq 0$ since $\bar{X}_{0}=0$. Because $e g v \in b_{1} \otimes X$ and $K[L]$-module $X$ is irreducible we have $K[(1, L, 1)] e g v=b_{1} \otimes X$. Then $K[M]$ egv $\supseteq K[G] b_{1} \otimes X=\bar{X}$. Hence $K[M] v=\bar{X}$. We have shown that $\bar{X}$ is an irreducible $K[M]$-module. This proves the assertion.

Note that the above theorem may be easily generalised to the case of arbitrary monoids of Lie type.

The next question is to decide when $\bar{X}$ is an irreducible $K[M]$-module. We shall see that the answer can be given in terms of group representation theory. First we need some preparatory observations.

It is easy to see, that $K[G]$-module $\bar{X}$ is isomorphic to the induced module $\operatorname{Ind}_{P}^{G}(X)$, where $K[P]$-module structure on $X$ comes from $\delta: P \rightarrow L$. Define $\xi: \bar{X} \rightarrow K[G / P] \otimes_{K[P]} X=\operatorname{Ind}_{P}^{G}(X)$ by $\xi\left(b_{i} \otimes x\right)=b_{i} \otimes x$ for $x \in X$ and extending by linearity. First we will show that $\xi$ is a homomorphism of $K[G]$-modules. Let $g \in G, x \in X$. Then $g\left(b_{i}, 1,1\right)=\left(b_{g(i)}, \delta(p(g, i)), 1\right)$ where $p(g, i) \in P$ is defined by $g b_{i}=b_{g(i)} p(g, i)$. Hence $g\left(b_{i} \otimes x\right)=b_{g(i)} \otimes \delta(p(g, i)) x$. This gives $\xi\left(g\left(b_{i} \otimes x\right)\right)=$ $\xi\left(b_{g(i)} \otimes \delta(p(g, i)) x\right)=b_{g(i)} \otimes \delta(p(g, i)) x$. Since also $g \xi\left(b_{i} \otimes x\right)=\left(g b_{i}\right) \otimes x=$ $\left(b_{g(i)} p(g, i)\right) \otimes x=b_{g(i)} \otimes(p(g, i) x)=b_{g(i)} \otimes \delta(p(g, i)) x$ then $\xi\left(g\left(b_{i} \otimes x\right)\right)=g \xi\left(b_{i} \otimes x\right)$. Since $\operatorname{Ind}_{P}^{G} X=\bigoplus_{i} b_{i} \otimes X, \xi$ is surjective. But $\operatorname{dim}_{K} \bar{X}=\operatorname{dim}_{K} \operatorname{Ind} d_{P}^{G} X$, so $\xi$ is an isomorphism. We can consider also $\operatorname{Ind}_{P^{-}}^{G} X$, where $K\left[P^{-}\right]$-module structure on $X$ comes from $\delta^{-}: P^{-} \rightarrow L$. Let us define the homomorphism of $K[G]$-modules

$$
\Phi=\operatorname{Ind}_{P, P^{-}}^{G}\left(i d_{X}\right): \operatorname{Ind}_{P^{-}}^{G} X \rightarrow \operatorname{Ind}_{P}^{G} X
$$

by

$$
\Phi(y)=\sum_{i=1}^{r} b_{i} \Phi_{1}\left(b_{i}^{-1} y\right) \text { for } y \in \operatorname{Ind}_{P^{-}}^{G} X
$$

where $\Phi_{1}: \operatorname{Ind}_{P^{-}}^{G} X \rightarrow X \subseteq \operatorname{Ind}_{P}^{G} X$ is given by:

$$
\begin{aligned}
& \Phi_{1}(p x)=p i d_{X}(x) \text { for } p \in P, x \in X, \\
& \Phi_{1}(g x)=0 \text { if } g \in G \backslash P P^{-}, x \in X .
\end{aligned}
$$

First we must show that $\Phi_{1}$ is well-defined. Assume that equality $p_{1} x_{1}=p_{2} x_{2}$ holds in $\operatorname{Ind}_{P^{-}}^{G} X$ for some $p_{1}, p_{2} \in P$ and $x_{1}, x_{2} \in X$. Then there exists $p^{-} \in P^{-}$ such that $p_{1}=p_{2} p^{-}$and $x_{2}=\delta^{-}\left(p^{-}\right) x_{1}$. Hence $p_{2}^{-1} p_{1}=p^{-} \in P \cap P^{-}$. The homomorphisms $\delta$ and $\delta^{-}$agree on $P \cap P^{-}$, so $\delta\left(p_{2}\right)^{-1} \delta\left(p_{1}\right)=\delta^{-}\left(p^{-}\right)$. Thus in Ind $d_{P}^{G} X$ we have: $p_{1} x_{1}=\delta\left(p_{1}\right) x_{1}=\delta\left(p_{2}\right) \delta^{-}\left(p^{-}\right) x_{1}=\delta\left(p_{2}\right) x_{2}=p_{2} x_{2}$. It is easy 
to see that $\Phi_{1}$ is a homomorphism of $K[P]$-modules. If $g \in G$, then $g^{-1} b_{i}=$ $b_{g(i)} p\left(g^{-1}, i\right)$ for some $p\left(g^{-1}, i\right) \in P$. Let $y \in \operatorname{Ind}_{P^{-}}^{G}$. Then

$$
\begin{gathered}
\Phi(g y)=\sum_{i=1}^{r} b_{i} \Phi_{1}\left(b_{i}^{-1} g y\right)=\sum_{i=1}^{r} b_{i} \Phi_{1}\left(p^{-1}\left(g^{-1}, i\right) b_{g(i)}^{-1} y\right) \\
=\sum_{i=1}^{r} b_{i} p^{-1}\left(g^{-1}, i\right) \Phi_{1}\left(b_{g(i)}^{-1} y\right)=\sum_{i=1}^{r} g b_{g(i)} \Phi_{1}\left(b_{g(i)}^{-1} y\right)=g \sum_{i=1}^{r} b_{i} \Phi_{1}\left(b_{i}^{-1} y\right)=g \Phi(y) .
\end{gathered}
$$

This shows that $\Phi$ is indeed a homomorphism of $K[G]$-modules. With the above notation we are now able to characterize the irreducibility of $\bar{X}$.

Theorem 2.2. Assume that $K$ is an algebraically closed field of characteristic zero and $\left|P^{-}\right| \geq|P|$. Then $\bar{X}$ is an irreducible $K[M]$-module if and only if $\operatorname{Ind}_{P, P^{-}}^{G}\left(i d_{X}\right)$ is an isomorphism.

Proof. Let $\langle\mid\rangle: X \times X \rightarrow K$ be an $L$-invariant, nonsingular bilinear form on $K$-linear space $X$. Define $\langle\mid\rangle: \bar{X} \times \bar{X} \rightarrow K$ by $\left\langle\sum_{i=1}^{r} b_{i} \otimes x_{i} \mid \sum_{i=1}^{r} b_{i} \otimes \bar{x}_{i}\right\rangle=$ $\sum_{i=1}^{r}\left\langle x_{i} \mid \bar{x}_{i}\right\rangle$. Then $\langle\mid\rangle$ is a $G$-invariant nonsingular bilinear form on $\bar{X}$. Let $\operatorname{ker}(e)=\{v \in \bar{X}: e v=0\}$. We claim that $\operatorname{ker} e \perp \xi^{-1} \Phi(X)$. Let $\sum_{i=1}^{r} b_{i} \otimes$ $x_{i} \in \operatorname{ker}(e), x_{i} \in X$. Since $e\left(b_{i}, 1,1\right)=\left(b_{1}, \gamma(e, i), 1\right)$, then $0=e \sum_{i=1}^{r} b_{i} \otimes x_{i}=$ $\sum_{i=1}^{r} b_{1} \otimes \gamma(e, i) x_{i}=b_{1} \otimes \sum_{i=1}^{r} \gamma(e, i) x_{i}$. So we have $\sum_{i=1}^{r} \gamma(e, i) x_{i}=0$ if and only if $\sum_{i=1}^{r} b_{i} \otimes x_{i} \in \operatorname{ker}(e)$.

Next, we will describe $\Phi(X)$. Let $x \in X$. Then by the definition $\Phi(x)=$ $\sum_{i=1}^{r} b_{i} \Phi_{1}\left(b_{i}^{-1} x\right)$. If $b_{i}^{-1}=p_{i}^{-1}\left(p_{i}^{-}\right)^{-1}, p_{i} \in P, p_{i}^{-} \in P^{-}$, then

$$
\begin{aligned}
b_{i} \Phi_{1}\left(b_{i}^{-1} x\right)= & b_{i} \Phi_{1}\left(p_{i}^{-1}\left(\delta^{-}\left(\left(p_{i}^{-}\right)^{-1}\right) x\right)\right)=b_{i} \delta\left(p_{i}^{-1}\right) \delta^{-}\left(\left(p_{i}^{-}\right)^{-1}\right) x \\
& =b_{i}\left[\delta^{-}\left(p_{i}^{-}\right) \delta\left(p_{i}\right)\right]^{-1} x=b_{i} \gamma(e, i)^{-1} x .
\end{aligned}
$$

On the other hand $b_{i} \Phi_{1}\left(b_{i}^{-1} x\right)=0$ if $b_{i} \notin P^{-} P$. Hence

$$
\Phi(x)=\sum_{i: \gamma(e, i) \neq 0} b_{i} \gamma(e, i)^{-1} x .
$$

This implies that

$$
\xi^{-1} \Phi(x)=\sum_{i: \gamma(e, i) \neq 0} b_{i} \otimes \gamma(e, i)^{-1} x .
$$

Let $\sum_{i=1}^{r} b_{i} \otimes x_{i} \in \operatorname{ker}(e), x_{i}, x \in X$. Then

$$
\begin{gathered}
\left\langle\sum_{i=1}^{r} b_{i} \otimes x_{i} \mid \xi^{-1} \Phi(x)\right\rangle=\left\langle\sum_{i=1}^{r} b_{i} \otimes x_{i} \mid \sum_{i: \gamma(e, i) \neq 0} b_{i} \otimes \gamma(e, i)^{-1} x\right\rangle \\
=\sum_{i: \gamma(e, i) \neq 0}\left\langle x_{i} \mid \gamma(e, i)^{-1} x\right\rangle=\sum_{i: \gamma(e, i) \neq 0}\left\langle\gamma(e, i) x_{i} \mid x\right\rangle=\left\langle\sum_{i=1}^{r} \gamma(e, i) x_{i} \mid x\right\rangle=\langle 0 \mid x\rangle=0 .
\end{gathered}
$$

Since $X$ is an irreducible $K[L]$-module, $\xi^{-1} \Phi(X)$ is $K[L]$-isomorphic to $X$ or 0 . If $\xi^{-1} \Phi(X)=0$ then $\Phi(g X)=0$ for all $g \in G$, so $\Phi=0$, a contradiction. This proves that the former holds. Hence $\operatorname{dim}_{K} \xi^{-1} \Phi(x)=\operatorname{dim}_{K} X$. Since $e \bar{X}$ and $X$ are $K[L]$-isomorphic, we have $\operatorname{dim}_{K} \operatorname{ker}(e)=\operatorname{dim}_{K} \bar{X}-\operatorname{dim}_{K} X$. Using the fact that $\xi^{-1} \Phi(X) \subseteq(\text { ker } e)^{\perp}$ and $\operatorname{dim}_{K} \xi^{-1} \Phi(X)+\operatorname{dim}_{K} \operatorname{ker}(e)=\operatorname{dim}_{K} \bar{X}$ we get $\xi^{-1} \Phi(X)=(\operatorname{ker} e)^{\perp}$. 
Now we are ready to prove the assertion of the theorem. Assume first that $\bar{X}$ is an irreducible $K[M]$-module. Let $v_{0} \in\left(\bigcap_{g \in G} g \operatorname{ker}(e)\right) \backslash\{0\}$. Then egv $=0$ for all $g \in G$. Hence $G e G v_{0}=0$. Define $Y=\{v \in \bar{X}: G e G v=0\}$. $Y$ is a $K[M]$-submodule of $\bar{X}$ since $G e G \cup\{0\}=J \cup\{0\}$ is an ideal of $M$. Hence $Y=\bar{X}$, so $e \bar{X}=0$. This contradiction proves that $\bigcap_{g \in G} g \operatorname{ker}(e)=0$. This implies that $\sum_{g \in G} g(\operatorname{ker} e)^{\perp}=\bar{X}$. Hence $\bar{X}=\sum_{g \in G} g \xi^{-1} \Phi(X)=\xi^{-1} \Phi\left(\sum_{g \in G} g X\right)=$ $\xi^{-1} \Phi\left(\operatorname{Ind}_{P^{-}}^{G} X\right)$ and $\Phi$ is surjective. Since also $\operatorname{dim}_{K} \operatorname{Ind}_{P^{-}}^{G} X \leq \operatorname{dim}_{K} \operatorname{Ind}_{P}^{G} X, \Phi$ is an isomorphism $\left(|P|=\left|P^{-}\right|\right.$in particular $)$. Conversely, suppose that $\Phi$ is an isomorphism. Thus $\bar{X}=\xi^{-1} \Phi\left(\operatorname{Ind}_{P^{-}}^{G} X\right)=\xi^{-1} \Phi\left(\sum_{g \in G} g X\right)=\sum_{g \in G} g \xi^{-1} \Phi(X)$. Hence $\bigcap_{g \in G} g \operatorname{ker}(e)=0$. Let $v \in \bar{X} \backslash\{0\}$. Then there exists $g \in G$ with $e g v \neq 0$. As at end of the proof of Theorem 2.1 this implies that the $K[M]$-module $\bar{X}$ is irreducible. This completes the proof.

\section{SEmisimplicity}

Let $G$ be a finite group. Then $G$ admits a BN-pair if there are subgroups $B, N$ of $G$ which generate $G$ such that $T=B \cap N \unlhd N$ and the Weyl group $W=N / T$ has a generating set of elements $s_{i}, i \in I$, with $s_{i}^{2}=1$ and

(i) $s_{i} B w \subseteq B s_{i} w B \cup B w B$ for every $s_{i}$ and $w \in W$,

(ii) $s_{i} B s_{i} \neq B$ for every $s_{i}$.

Then $W$ is a Coxeter group. If $w \in W$, we define $l(w)$ to be the minimal length of $w$ as a product of the generators $s_{i}, i \in I$. In particular $l(w)=0$ if and only if $w=1$. The conjugates of $B$ are called Borel subgroups. Any subgroup $P$ of $G$ containing a Borel subgroup is called a parabolic subgroup of $G$. Let $W_{J}$ be the subgroup of $W$ generated by elements $s_{i}$ with $i \in J$ for some $J \subseteq I$. Then $P_{J}$ denotes the standard parabolic subgroup $B W_{J} B$. Any parabolic subgroup $P$ of $G$ is conjugate to a unique $P_{J}$. It turns out that $W_{J}$ has a unique element $\left(w_{0}\right)_{J}$ of maximal length. Then $\left(w_{0}\right)_{J}$ is of order 2. Write $\left(w_{0}\right)_{I}=w_{0}$ and $B^{-}=w_{0} B w_{0}$. Then $P_{J}^{-}=B^{-} W_{J} B^{-}$is a parabolic subgroup of $G$ which is called opposite to $P_{J}$. We will assume as in [1, chapter 2], that $G$ admits a split B-N pair satisfying some commutator relations. Then there exists a normal subgroup $U$ of $B$ such that $B=U T, U \cap T=\{1\}$. Let $\Phi$ be the root system of $W$ and $\Delta=\left\{\alpha_{1}, \alpha_{2}, \ldots, \alpha_{l}\right\} \subseteq \Phi(l=|I|)$ the set of simple roots. Each root $\alpha \in \Phi$ has the form $\alpha=\sum_{i=1}^{l} \lambda_{i} \alpha_{i}$ where either all $\lambda_{i} \geq 0$ or all $\lambda_{i} \leq 0$. Roots with all $\lambda_{i} \geq 0$ are called positive roots $(\alpha>0)$ and their set is denoted by $\Phi^{+}$. Let $\Delta_{J}=\left\{\alpha_{i}: i \in J\right\}(J \subseteq I)$. Define $\Phi_{J}=W_{J}\left(\Delta_{J}\right)$. Then $\Phi_{J}$ is a root system for $W_{J}$ with the set of simple roots $\Delta_{J}$. We also define subgroups of $G$ : $U^{-}=U^{w_{0}}, X_{i}=U \cap U^{w_{0} s_{i}}, U_{w}=U \cap U^{w_{0} w}$ for $w \in W$. If $w\left(\alpha_{i}\right)=\alpha, w \in W, \alpha \in \Phi$, we will denote the root subgroup $w X_{i} w^{-1}$ by $X_{\alpha}$. The definition is independent of the choice of $w$ and $\alpha_{i}$. Then $U_{w}$ is a product of $X_{\beta}$ such that $\beta>0$ and $w(\beta)<0$. Let $U_{J}=U \cap U^{\left(w_{0}\right)_{J}}, U_{J}^{-}=U \cap U^{w_{0}\left(w_{0}\right)_{J}}, L_{J}=\left\langle T, X_{\alpha}: \alpha \in \Phi_{J}\right\rangle\left(=P_{J} \cap P_{J}^{-}\right)$. Then $U_{J}, U_{J}^{-}$are normal subgroups of $P_{J}, P_{J}^{-}$respectively and we have the Levi decompositions $P_{J}=U_{J} L_{J}, P_{J}^{-}=U_{J}^{-} L_{J}$ with $U_{J} \cap L_{J}=\{1\}, U_{J}^{-} \cap L_{J}=\{1\}$. $L_{J}$ is called a Levi factor of $P_{J}$. Any Levi factor of $P_{J}$ is conjugate to $L_{J}$ by some element of $U_{J} . U_{J}, U_{J}^{-}$are called unipotent radicals of $P_{J}, P_{J}^{-}$respectively. $U_{J}$ is the product of $X_{\beta}$ with $\beta>0$ and $\beta \notin \Phi_{J}$ in any order. Let $J_{1}, J_{2} \subseteq I$. We define $D_{J_{1}, J_{2}}=\left\{w \in W: w^{-1}\left(\Delta_{J_{1}}\right)>0\right.$ and $\left.w\left(\Delta_{J_{2}}\right)>0\right\}$. Then $W=W_{J_{1}} D_{J_{1}, J_{2}} W_{J_{2}}$ and any $w \in D_{J_{1}, J_{2}}$ is the unique element of minimal length in $W_{J_{1}} w W_{J_{2}}$. Moreover we have $G=\bigsqcup_{w \in D_{J_{1}, J_{2}}} P_{J_{1}} w P_{J_{2}}$. See [1, chapter 2], for details. 
Next we restrict our attention to monoids $M=M\left(G, P, P^{-}, L\right)$ where $G$ is a finite group with a split BN-pair satisfying some commutator relations and $P, P^{-}$are parabolic subgroups of $G$ with a common Levi factor $L$. Then $P=U L, P^{-}=U^{-} L$ where $U, U^{-}$are the unipotent radicals of $P, P^{-}$respectively. Homomorphisms $\delta$ and $\delta^{-}$are defined by $\delta(u l)=l, \delta^{-}\left(u^{-} l\right)=l$ for $l \in L, u \in U, u^{-} \in U^{-}$. The monoid defined by $\left(g P g^{-1}, g L g^{-1}, g P^{-} g^{-1}\right), g \in G$, is isomorphic to that defined by $\left(P, L, P^{-}\right)$. Let us write $\left(P, L, P^{-}\right) \sim\left(g P g^{-1}, g L g^{-1}, g P^{-} g^{-1}\right)$ in this case. We will find $g \in G$ such that $\left(g P g^{-1}, g L g^{-1}, g P^{-} g^{-1}\right)$ is 'standard'. There exist $g_{1}, g_{2} \in G$ and $J_{1}, J_{2} \subseteq I$ with $P=P_{J_{1}}^{g_{1}}, P^{-}=P_{J_{2}}^{g_{2}}$. Since $G=P_{J_{1}} D_{J_{1}, J_{2}} P_{J_{2}}$, then $g_{1} g_{2}^{-1}=p_{1} w p_{2}$ where $p_{i} \in P_{J_{i}}, w \in D_{J_{1}, J_{2}}$. Thus

$$
\begin{gathered}
\left(P, L, P^{-}\right) \sim\left(P_{J_{1}}, L^{g_{1}^{-1}}, P_{J_{2}}^{g_{2} g_{1}^{-1}}\right) \sim\left(P_{J_{1}}, L^{g_{1}^{-1}}, P_{J_{2}}^{p_{2}^{-1} w^{-1} p_{1}^{-1}}\right) \\
\sim\left(P_{J_{1}}^{p_{1}}, L^{g_{1}^{-1} p_{1}},\left(P_{J_{2}}^{p_{2}^{-1}}\right)^{w^{-1}}\right)=\left(P_{J_{1}}, \bar{L}, P_{J_{2}}^{w^{-1}}\right) .
\end{gathered}
$$

So we can assume that $P=P_{J_{1}}, P^{-}=P_{J_{2}}^{w^{-1}}, w \in D_{J_{1}, J_{2}}$. Next $L=u_{1} L_{J_{1}} u_{1}^{-1}=$ $\left(u_{2} L_{J_{2}} u_{2}^{-1}\right)^{w^{-1}}$ for some $u_{i} \in U_{J_{i}}$. By [1, Prop.2.8.3] $P_{J_{1}} \cap P_{J_{2}}^{w^{-1}} \subseteq P_{K}$ where $\Delta_{K}=\Delta_{J_{1}} \cap w\left(\Delta_{J_{2}}\right)$. Hence $u_{1} L_{J_{1}} u_{1}^{-1} \subseteq P_{K}$. Since $U_{J_{1}} \subseteq U_{K}$, then $L_{J_{1}} \subseteq P_{K}$. This gives $P_{J_{1}}=U_{J_{1}} L_{J_{1}} \subseteq P_{K}$. So $J_{1} \subseteq K$ and $\Delta_{J_{1}} \subseteq w\left(\Delta_{J_{2}}\right)$ by the definition of $K$. Similar considerations applied to $P_{J_{2}} \cap P_{J_{1}}^{w}\left(w^{-1} \in D_{J_{2}, J_{1}}\right)$ allow us to prove that $\Delta_{J_{2}} \subseteq w^{-1}\left(\Delta_{J_{1}}\right)$. Hence $\Delta_{J_{1}}=w\left(\Delta_{J_{2}}\right)$. By [1, Th.2.8.7]

$$
P_{J_{1}} \cap P_{J_{2}}^{w^{-1}}=\left(U_{J_{1}} \cap U_{J_{2}}^{w^{-1}}\right)\left(U_{J_{1}} \cap L_{J_{2}}^{w^{-1}}\right)\left(L_{J_{1}} \cap U_{J_{2}}^{w^{-1}}\right) L_{K}
$$

where $\Delta_{K}=\Delta_{J_{1}} \cap w\left(\Delta_{J_{2}}\right)$. Since $\Delta_{J_{1}}=w\left(\Delta_{J_{2}}\right)$, we have $K=J_{1}$. By [1, Cor.2.8.8] $U_{J_{1}} \cap L_{J_{2}}^{w^{-1}}=\{1\}$ and $L_{J_{1}} \cap U_{J_{2}}^{w^{-1}}=\{1\}$. Hence $P_{J_{1}} \cap P_{J_{2}}^{w^{-1}}=\left(U_{J_{1}} \cap U_{J_{2}}^{w^{-1}}\right) L_{J_{1}}=$ $\left(U_{J_{1}} \cap U_{J_{2}}^{w^{-1}}\right) L$. This proves that $\delta$ and $\delta^{-}$agree on $P_{J_{1}} \cap P_{J_{2}}^{w^{-1}}$.

Next we will show that $U_{J_{2}}^{w^{-1}}=\left(U_{J_{1}} \cap U_{J_{2}}^{w^{-1}}\right)\left(U_{J_{1}}^{-} \cap U_{J_{2}}^{w^{-1}}\right)$. Since $U_{J_{2}}^{w^{-1}}$ is a product of $X_{\alpha}^{w^{-1}}$, where $\alpha>0$ and $\alpha \notin \Phi_{J_{2}}$, in any order, it is sufficient to show that $X_{\alpha}^{w^{-1}} \subseteq U_{J_{1}} \cap U_{J_{2}}^{w^{-1}}$ or $X_{\alpha}^{w^{-1}} \subseteq U_{J_{1}}^{-} \cap U_{J_{2}}^{w^{-1}}$. We see that $w(\alpha) \notin$ $w\left(\Phi_{J_{2}}\right)=\Phi_{J_{1}}$. Hence, if $w(\alpha)>0$, then $X_{\alpha}^{w^{-1}}=X_{w(\alpha)} \subseteq U_{J_{1}} \cap U_{J_{2}}^{w^{-1}}$ and, if $w(\alpha)<0$, then $X_{w(\alpha)} \subseteq U_{J_{1}}^{-} \cap U_{J_{2}}^{w^{-1}}$. So we can factorize $u_{2}^{w^{-1}}$ as $u_{2}^{w^{-1}}=\bar{u}_{1} \overline{\bar{u}}_{1}$ where $\bar{u}_{1} \in U_{J_{1}} \cap U_{J_{2}}^{w^{-1}}, \overline{\bar{u}}_{1} \in U_{J_{1}}^{-} \cap U_{J_{2}}^{w^{-1}}$. Thus

$$
\bar{u}_{1}^{-1} u_{1} L_{J_{1}} u_{1}^{-1} \bar{u}_{1}=\overline{\bar{u}}_{1} L_{J_{1}} \overline{\bar{u}}_{1}^{-1} \subseteq P_{J_{1}} \cap P_{J_{1}}^{-}=L_{J_{1}} .
$$

So $L_{J_{1}}=\bar{u}_{1}^{-1} u_{1} L_{J_{1}} u_{1}^{-1} \bar{u}_{1}$. This gives $L=\bar{u}_{1} L_{J_{1}} \bar{u}_{1}^{-1}$. Here $\bar{u}_{1} \in U_{J_{1}} \cap U_{J_{2}}^{w^{-1}}$. Now we have

$$
\left(P_{J_{1}}, L, P_{J_{2}}^{w^{-1}}\right) \sim\left(P_{J_{1}}, L_{J_{1}}^{\bar{u}_{1}^{-1}}, P_{J_{2}}^{w^{-1}}\right) \sim\left(P_{J_{1}}^{\bar{u}_{1}}, L_{J_{1}}, P_{J_{2}}^{w^{-1} \bar{u}_{1}}\right)=\left(P_{J_{1}}, L_{J_{1}}, P_{J_{2}}^{w^{-1}}\right) .
$$

So there is no loss of generality in assuming that $P=P_{J_{1}}, L=L_{J_{1}}, P^{-}=P_{J_{2}}^{w^{-1}}$ and $w\left(\Delta_{J_{2}}\right)=\Delta_{J_{1}}$ (this implies that $\left.w \in D_{J_{1}, J_{2}}\right)$.

Let $K_{1}$ satisfy $K_{1} \subseteq J_{1} . K_{2}$ is defined by $w\left(\Delta_{K_{2}}\right)=\Delta_{K_{1}}$. Clearly $K_{2} \subseteq J_{2}$. Let $Y$ be a $K\left[L_{K_{1}}\right]$-module. Consider the homomorphism of $K[G]$-modules

$$
\phi=\operatorname{Ind}_{P_{K_{1}}, P_{K_{2}}^{w-1}}^{G}\left(i d_{Y}\right): \operatorname{Ind}_{P_{K_{2}}^{w-1}}^{G} Y \rightarrow \operatorname{Ind}_{P_{K_{1}}}^{G} Y
$$

defined as before Theorem $2.2\left(K\left[P_{K_{2}}^{w^{-1}}\right]\right.$-structure on $Y$ is determined by the trivial action of $U_{K_{2}}^{w^{-1}}$ and similarly $K\left[P_{K_{1}}\right]$-structure by $U_{K_{1}}$ acting trivially). $P_{K_{1}} \cap L_{J_{1}}$ 
is a standard parabolic subgroup of $L_{J_{1}}$ and we have the Levi decomposition $P_{K_{1}} \cap$ $L_{J_{1}}=\left(U_{K_{1}} \cap L_{J_{1}}\right) L_{K_{1}}$, cf.[1, Prop.2.8.9]. Similarly we define

$$
\bar{\phi}=\operatorname{Ind}_{P_{K_{1}} \cap L_{J_{1}},\left(P_{K_{2}} \cap L_{J_{2}}\right)^{w^{-1}}}^{L_{J_{1}}}\left(i d_{Y}\right): \operatorname{Ind}_{\left(P_{K_{2}} \cap L_{J_{2}}\right)^{w^{-1}}}^{L_{J_{2}}^{w^{-1}}} Y \rightarrow \operatorname{Ind}_{P_{K_{1}} \cap L_{J_{1}}}^{L_{J_{1}}} Y
$$

(here $\left(U_{K_{2}} \cap L_{J_{2}}\right)^{w^{-1}}, U_{K_{1}} \cap L_{J_{1}}$ act trivially on suitable copies of $Y$ ). Finally, we define

$$
\operatorname{Ind}_{P_{J_{1}}, P_{J_{2}}^{w^{-1}}}^{G}(\bar{\phi}): \operatorname{Ind}_{P_{J_{2}}^{w-1}}^{G}\left(\operatorname{Ind}_{\left(P_{K_{2}} \cap L_{J_{2}}\right)^{w^{-1}}}^{L_{J_{2}}^{w-1}} Y\right) \rightarrow \operatorname{Ind}_{P_{J_{1}}}^{G}\left(\operatorname{Ind}_{P_{K_{1}} \cap L_{J_{1}}}^{L J_{1}} Y\right)
$$

(where $U_{J_{2}}^{w^{-1}}$ acts as 1 on $\operatorname{Ind} d_{\left(P_{K_{2}} \cap L_{J_{2}}\right)^{-1}}^{L_{J_{2}}^{-1}} Y, U_{J_{1}}$ acts as 1 on $\operatorname{Ind}_{P_{K_{1}} \cap L_{J_{1}}}^{L_{J_{1}}} Y$ ). Notice that $\operatorname{Ind}_{P_{J_{1}}}^{G} \operatorname{Ind}_{P_{K_{1}} \cap L_{J_{1}}}^{L_{J_{1}}} Y=\operatorname{Ind}_{P_{K_{1}}}^{G} Y$. The following lemma is an analogue of this equality for Ind homomorphisms.

Lemma 3.1. With the above notation we have

$$
\operatorname{Ind}_{P_{K_{1}, P_{K_{2}}}^{w^{-1}}}^{G}\left(i d_{Y}\right)=\operatorname{Ind} d_{P_{J_{1}}, P_{J_{2}}^{w-1}}^{G}\left(\operatorname{Ind}_{P_{K_{1}} \cap L_{J_{1}},\left(P_{K_{2}} \cap L_{J_{2}}\right)^{w^{-1}}}^{L_{J_{1}}}\left(i d_{Y}\right)\right) .
$$

Proof. By the definition of homomorphisms of type Ind we have

$$
\left(\operatorname{Ind}_{P_{J_{1}}, P_{J_{2}}^{w-1}}^{G}(\bar{\phi})\right)_{1}\left(p_{J_{1}} \bar{y}\right)=p_{J_{1}} \bar{\phi}(\bar{y}) \text { for } p_{J_{1}} \in P_{J_{1}}, \bar{y} \in \operatorname{Ind}_{\left(P_{K_{2}} \cap L_{J_{2}}\right)^{w^{-1}}}^{L_{J_{1}}^{w^{-1}} Y .}
$$

Let $p_{K_{1}} \in P_{K_{1}}$. Then $p_{K_{1}}=u_{J_{1}} l_{J_{1}}$ for some $u_{J_{1}} \in U_{J_{1}}, l_{J_{1}} \in L_{J_{1}}$. Since $u_{J_{1}} \in U_{K_{1}}$, we have $l_{J_{1}} \in P_{K_{1}} \cap L_{J_{1}}$. Moreover, let $y \in Y$. Then

$$
\begin{gathered}
\left(\left(\operatorname{Ind}_{P_{J_{1}}, P_{J_{2}}^{w-1}}^{G}(\bar{\phi})\right)_{1}\right)_{1}\left(p_{K_{1}} y\right)=\left(p_{K_{1}} \bar{\phi}(y)\right)_{1}=\left(u_{J_{1}} l_{J_{1}} \bar{\phi}(y)\right)_{1}=\left(l_{J_{1}} \bar{\phi}(y)\right)_{1} \\
=\left(\bar{\phi}\left(l_{J_{1}} y\right)\right)_{1}=\bar{\phi}_{1}\left(l_{J_{1}} y\right)=l_{J_{1}} y=u_{J_{1}} l_{J_{1}} y=p_{K_{1}} y=\left(\operatorname{Ind}_{P_{K_{1}}, P_{K_{2}}^{w-1}}^{G}\left(i d_{Y}\right)\right)_{1}\left(p_{K_{1}} y\right) .
\end{gathered}
$$

Next we prove that $\left(\left(\operatorname{Ind}_{P_{J_{1}}, P_{J_{2}}^{w-1}}^{G}(\bar{\phi})\right)_{1}\right)_{1}(g y)=0=\left(\operatorname{Ind}_{P_{K_{1}}, P_{K_{2}}^{w-1}}^{G}\left(i d_{Y}\right)\right)_{1}(g y)$ for $g \notin P_{K_{1}} P_{K_{2}}^{w^{-1}}$ and $y \in Y$. The second equality follows from the definition. The first equality holds if $g \notin P_{J_{1}} P_{J_{2}}^{w^{-1}}$ since $\left(\operatorname{Ind}_{P_{J_{1}}, P_{J_{2}}^{w-1}}^{G}(\bar{\phi})\right)_{1}(g y)=0$. So we can assume that $g=p_{J_{1}} p_{J_{2}}^{w^{-1}}$ for some $p_{J_{i}} \in P_{J_{i}}$. Moreover, let $p_{J_{1}}=u_{J_{1}} l_{J_{1}}, p_{J_{2}}=u_{J_{2}} l_{J_{2}}$ where $u_{J_{i}} \in U_{J_{i}}, l_{J_{i}} \in L_{J_{i}}$. Then we have

$$
\begin{gathered}
\left(\left(\operatorname{Ind}_{P_{J_{1}}, P_{J_{2}}^{w-1}}^{G}(\bar{\phi})\right)_{1}\right)_{1}(g y)=\left[\left(\operatorname{Ind}_{P_{J_{1}}, P_{J_{2}}^{w-1}}^{G}(\bar{\phi})\right)_{1}\left(p_{J_{1}} u_{J_{2}}^{w^{-1}}\left(l_{J_{2}}^{w^{-1}} y\right)\right)\right]_{1} \\
=\left[\left(\operatorname{Ind}_{P_{J_{1}}, P_{J_{2}}^{w-1}}^{G}(\bar{\phi})\right)_{1}\left(p_{J_{1}}\left(l_{J_{2}}^{w^{-1}} y\right)\right)\right]_{1}=\left(p_{J_{1}} \bar{\phi}\left(l_{J_{2}}^{w^{-1}} y\right)\right)_{1}=\left(u_{J_{1}} l_{J_{1}} \bar{\phi}\left(l_{J_{2}}^{w^{-1}} y\right)\right)_{1} \\
=\left(l_{J_{1}} \bar{\phi}\left(l_{J_{2}}^{w^{-1}} y\right)\right)_{1}=\left(\bar{\phi}\left(l_{J_{1}} l_{J_{2}}^{w^{-1}} y\right)\right)_{1}=\bar{\phi}_{1}\left(l_{J_{1}} l_{J_{2}}^{w^{-1}} y\right) .
\end{gathered}
$$

Since $u_{J_{1}} l_{J_{1}} u_{J_{2}}^{w^{-1}} l_{J_{2}}^{w^{-1}} \notin P_{K_{1}} P_{K_{2}}^{w^{-1}}$ by the assumption, then

$$
l_{J_{1}} l_{J_{2}}^{w^{-1}} \notin\left(u_{J_{1}}^{-1} P_{K_{1}}\right)\left(P_{K_{2}}\left(u_{J_{2}}^{-1}\right)^{l_{J_{2}}}\right)^{w^{-1}}=P_{K_{1}} P_{K_{2}}^{w^{-1}} .
$$

This implies $l_{J_{1}} l_{J_{2}}^{w^{-1}} \notin\left(P_{K_{1}} \cap L_{J_{1}}\right)\left(P_{K_{2}} \cap L_{J_{2}}\right)^{w^{-1}}$. Hence

$$
\left(\left(\operatorname{Ind}_{P_{J_{1}}, P_{J_{2}}^{w-1}}^{w^{-1}}(\bar{\phi})\right)_{1}\right)_{1}(g y)=\bar{\phi}_{1}\left(l_{J_{1}} l_{J_{2}}^{w^{-1}} y\right)=0 .
$$

We have proved that

$$
\left(\left(\operatorname{Ind}_{P_{J_{1}}, P_{J_{2}}^{w-1}}^{G}(\bar{\phi})\right)_{1}\right)_{1}(g y)=\left(\operatorname{Ind}_{P_{K_{1}}, P_{K_{2}}^{w-1}}^{G}\left(i d_{Y}\right)\right)_{1}(g y)
$$


for $y \in Y, g \in P_{K_{1}}$ and also for $g \notin P_{K_{1}} P_{K_{2}}^{w^{-1}}$. So $\left(\left(\operatorname{Ind}_{P_{J_{1}}, P_{J_{2}}^{w-1}}^{G}(\bar{\phi})\right)_{1}\right)_{1}=$ $\left(\operatorname{Ind}_{P_{K_{1}}, P_{K_{2}}^{w-1}}^{G}\right)_{1}$. This establishes the desired formula.

We are now ready for the main result of this section.

Theorem 3.2. Assume that $M=M\left(G, P, P^{-}, L\right)$ where $G$ is a finite group with a split BN-pair satisfying some commutator relations and $P, P^{-}$are parabolic subgroups of $G$ with a common Levi factor $L$. Let $K$ be an algebraically closed field of characteristic zero. Then the semigroup algebra $K_{0}[M]$ is semisimple.

Proof. By [3] we can assume that $K$ is the field of complex numbers. We know, that it is sufficient to prove the theorem for $P=P_{J_{1}}, L=L_{J_{1}}, P^{-}=P_{J_{2}}^{w^{-1}}$ where $w\left(\Delta_{J_{2}}\right)=\Delta_{J_{1}}$. By the symmetry of our problem it may be assumed that $\left|P^{-}\right| \geq|P|$. By Theorem 2.1 it suffices to prove that all $K[M]$-modules $\bar{X}$, where $X$ runs over the set of all irreducible $K\left[L_{J_{1}}\right]$-modules, are irreducible. Fix some $X$. In view of Theorem 2.2 this is equivalent to $\operatorname{Ind}_{P_{J_{1}}, P_{J_{2}}^{w^{-1}}}^{G}\left(i d_{X}\right)$ being an isomorphism of $K[G]$-modules. Suppose that the representation of $L_{J_{1}}$ in $E n d_{K} X$ is cuspidal. This means that the $K\left[L_{J_{1}}\right]$-module $\mathrm{X}$ is not a component of any induced module Ind $_{P_{K_{1}} \cap L_{J_{1}}}^{L_{J_{1}}} Y$, where $K_{1} \subset J_{1}, Y$ is a $K\left[K_{1}\right]$-module and $U_{K_{1}} \cap L_{J_{1}}$ acts trivially on $Y$. As in $[1,10.1]$ we will consider a functional representation of $\operatorname{Ind}_{P_{J_{1}}}^{G} X$. Let $\rho: L_{J_{1}} \rightarrow \operatorname{End}_{K} X$ be the associated $K$-representation. Consider the set $\mathcal{F}$ of all maps from $G$ to $X$. $\mathcal{F}$ may be made into a left $K[G]$-module by $(g f)(x)=f(x g)$ where $x, g \in G, f \in \mathcal{F}$. Let $\mathcal{F}\left(J_{1}, \rho\right)$ be the subset of $\mathcal{F}$ defined by

$$
\mathcal{F}\left(J_{1}, \rho\right)=\left\{f \in \mathcal{F}: f(p g)=p f(g) \text { for } p \in P_{J_{1}}, g \in G\right\} .
$$

By [1, Prop.10.1.1] $K[G]$-modules $\mathcal{F}\left(J_{1}, \rho\right)$ and $\operatorname{Ind}_{P_{J_{1}}}^{G} X$ are isomorphic. Let us define a $K[G]$-homomorphism $\tau_{1}: \mathcal{F}\left(J_{1}, \rho\right) \rightarrow \operatorname{Ind}_{P_{J_{1}}}^{G} X$ by $\tau_{1}(f)=\sum_{i=1}^{r} g_{i}^{-1} f\left(g_{i}\right)$ where $f \in \mathcal{F}\left(J_{1}, \rho\right)$ and $G=\bigsqcup_{i=1}^{r} P_{J_{1}} g_{i}$ with $g_{1}=1$. In fact $\tau_{1}$ is an isomorphism, cf. [1, proof of Prop.10.1.1]. The inverse homomorphism is determined by $\tau_{1}^{-1}(x)=$ $f_{x}, x \in X$, where $f_{x}$ satisfies $f_{x}(p)=p x$ for $p \in P_{J_{1}}$ and $f_{x}(g)=0$ for $g \notin P_{J_{1}}$. Similarly we can define a $K[G]$-isomorphism $\tau_{2}: \mathcal{F}\left(J_{2}, \rho^{w}\right) \rightarrow \operatorname{Ind}_{P_{J_{2}}}^{G}\left(w^{-1} X\right)$. We have also an obvious $K[G]$-isomorphism $\tau^{\prime}: \operatorname{Ind}_{P_{J_{2}}^{w-1}}^{G} X \rightarrow \operatorname{Ind}_{P_{J_{2}}}^{G}\left(w^{-1} X\right)$ given by $\tau^{\prime}(x)=x$ for $x \in X$. Let $\bar{\tau}=\tau_{2}^{-1} \tau^{\prime}$. Finally, consider the $K[G]$-homomorphism $\theta_{w}: \mathcal{F}\left(J_{2}, \rho^{w}\right) \rightarrow \mathcal{F}\left(J_{1}, \rho\right)$ defined by $\theta_{w}(f) g=\left|U_{J_{1}}\right|^{-1} \sum_{u \in U_{J_{1}}} f\left(w^{-1} u g\right)$ for any $g \in G$, see [1, Prop.10.1.3]. We get the following diagram of $K[G]$-homomorphisms.

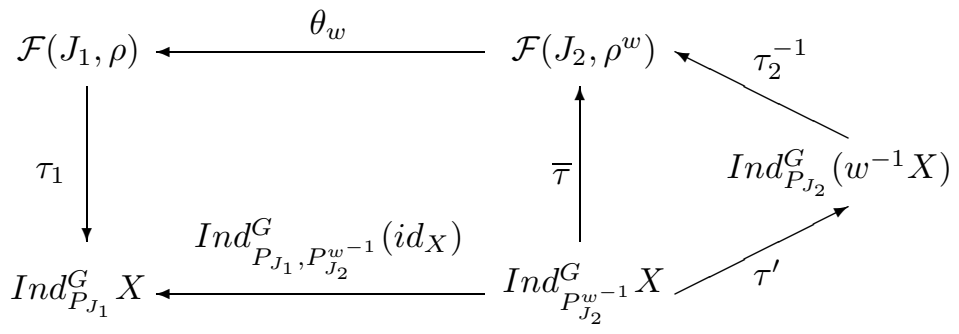


Next we will establish a link between $\theta_{w}$ and $\operatorname{Ind}_{P_{J_{1}}, P_{J_{2}}^{w-1}}^{G}\left(i d_{X}\right)$. We claim, that

$$
\tau_{1} \theta_{w} \bar{\tau}=\frac{\left|U_{J_{1}} \cap U_{J_{2}}^{w^{-1}}\right|}{\left|U_{J_{1}}\right|} \operatorname{Ind} d_{P_{J_{1}}, P_{J_{2}}^{w-1}}^{G}\left(i d_{X}\right) .
$$

Suppose $g \in G, x \in X$. Then we have

$$
\begin{gathered}
\left(\tau_{1} \theta_{w} \bar{\tau}\right)_{1}(g x)=\left[\tau_{1} \theta_{w} \bar{\tau}\left((g w)\left(w^{-1} x\right)\right)\right]_{1}=\left[\tau_{1} \theta_{w}\left(g w \bar{\tau}\left(w^{-1} x\right)\right)\right]_{1} \\
=\left[\tau_{1}\left(\theta_{w}\left(g w f_{x}\right)\right)\right]_{1}=\theta_{w}\left(g w f_{x}\right)(1)=\left|U_{J_{1}}\right|^{-1} \sum_{u \in U_{J_{1}}} g w f_{x}\left(w^{-1} u\right) \\
=\left|U_{J_{1}}\right|^{-1} \sum_{u \in U_{J_{1}}} f_{x}\left(w^{-1} u g w\right) .
\end{gathered}
$$

If $f_{x}\left(w^{-1} u g w\right) \neq 0$, then $u g \in P_{J_{2}}^{w^{-1}}$. Hence $g \in P_{J_{1}} P_{J_{2}}^{w^{-1}}$. This gives $\left(\tau_{1} \theta_{w} \bar{\tau}\right)_{1}(g x)$ $=0$ if $g \notin P_{J_{1}} P_{J_{2}}^{w^{-1}}$. Next, let $g=p_{1} \in P_{J_{1}}$. Then $p_{1}=u_{1} l_{1}$ with $u_{1} \in U_{J_{1}}, l_{1} \in$ $L_{J_{1}}$. Hence $f_{x}\left(w^{-1} u g w\right) \neq 0$ implies that $u u_{1} l_{1} \in P_{J_{2}}^{w^{-1}}$. Therefore $u u_{1} \in P_{J_{2}}^{w^{-1}} \cap$ $U_{J_{1}}=U_{J_{2}}^{w^{-1}} \cap U_{J_{1}}$, the equality following from [1, Prop.2.8.6 and Cor.2.8.8] since $w\left(\Delta_{J_{2}}\right)=\Delta_{J_{1}}$. So we have $f_{x}\left(w^{-1} u g w\right)=u u_{1}\left(l_{1} x\right)=l_{1} x$ since $u u_{1} \in U_{J_{2}}^{w^{-1}}$. In this case $\left(\tau_{1} \theta_{w} \bar{\tau}\right)_{1}(g x)=\left|U_{J_{1}} \cap U_{J_{2}}^{w^{-1}}\right|\left|U_{J_{1}}\right|^{-1} l_{1} x$. We have shown that $\left(\tau_{1} \theta_{w} \bar{\tau}\right)_{1}=$ $\left|U_{J_{1}} \cap U_{J_{2}}^{w^{-1}}\right|\left|U_{J_{1}}\right|^{-1}\left[\operatorname{Ind}_{P_{J_{1}}, P_{J_{2}}^{w}}^{G}\left(i d_{X}\right)\right]_{1}$. This gives (2). According to (2), in order to show that $\operatorname{Ind}_{P_{J_{1}}, P_{J_{2}}^{w-1}}^{G}\left(i d_{X}\right)$ is an isomorphism, it is sufficient to prove that $\theta_{w}$ is an isomorphism. This was done in the last part of the proof of [1, Prop.10.7.9].

Finally, assume that the representation of $L_{J_{1}}$ in $E n d_{K} X$ is not cuspidal. Then $K\left[L_{J_{1}}\right]$-module $X$ is a component of $\operatorname{Ind}_{P_{K_{1}} \cap L_{J_{1}}}^{L_{J_{1}}} Y$ where $K_{1} \subset J_{1}, Y$ is an irreducible $K\left[L_{K_{1}}\right]$-module whose associated representation is cuspidal and $U_{K_{1}} \cap L_{J_{1}}$ acts trivially on $Y$. We know that $\bar{\Phi}=\operatorname{Ind}_{P_{K_{1}} \cap L_{J_{1}},\left(P_{K_{2}} \cap L_{J_{2}}\right)^{w^{-1}}}\left(i d_{Y}\right)$ is an isomorphism. Since $\left.\bar{\Phi}\right|_{\bar{\Phi}^{-1}(X)}: \bar{\Phi}^{-1}(X) \rightarrow X$ is a component of $\bar{\Phi}$ then $\operatorname{Ind}_{P_{J_{1}}, P_{J_{2}}^{w-1}}^{G}\left(\left.\bar{\Phi}\right|_{\Phi^{-1}(X)}\right)$ is a component of $\operatorname{Ind}_{P_{J_{1}}, P_{J_{2}}^{w-1}}^{G}(\bar{\Phi})$ which is equal to Ind $d_{P_{K_{1}}, P_{K_{2}}^{w-1}}^{G}\left(i d_{Y}\right)$ by Lemma 3.1. The cuspidal case implies that $\operatorname{Ind}_{P_{K_{1}}, P_{K_{2}}^{w-1}}^{G}\left(i d_{Y}\right)$ is an isomorphism. Therefore $\operatorname{Ind}_{P_{J_{1}}, P_{J_{2}}^{w}}^{G}\left(\left.\bar{\Phi}\right|_{\Phi^{-1}(X)}\right)$ is an isomorphism. But $\operatorname{Ind}_{P_{J_{1}}, P_{J_{2}}^{w-1}}^{G}\left(\left.\bar{\Phi}\right|_{\Phi^{-1}(X)}\right) \simeq \operatorname{Ind}_{P_{J_{1}}, P_{J_{2}}^{w-1}}^{G}\left(i d_{X}\right)$, hence also $\operatorname{Ind}_{P_{J_{1}}, P_{J_{2}}^{w}}^{G}\left(i d_{X}\right)$ is an isomorphism. This completes the proof.

The above theorem yields in particular a new proof of the semisimplicity of $\mathbf{C}_{0}[M]$ for monoids of Lie type $M$, originally proved in [6].

\section{DeCOMPOSING SANDWICH MATRICES}

Let $S=\left(s_{j, i}\right)$ be the sandwich matrix of the monoid defined by $\left(P_{J_{1}}, L_{J_{1}}, P_{J_{2}}^{w_{1}}\right)$, where $w_{1} \in W, w_{1}\left(\Delta_{J_{1}}\right)=\Delta_{J_{2}}$, with respect to the following coset decompositions $G=\bigsqcup_{i} b_{i} P_{J_{1}}=\bigsqcup_{j} P_{J_{2}}^{w_{1}} a_{j}$. Let $\bar{S}=\left(\bar{s}_{k, j}\right)$ be the sadwich matrix of $\left(P_{J_{2}}^{w_{1}}, L_{J_{1}}, P_{J_{3}}^{w_{2} w_{1}}\right)$ where $w_{2} \in W, w_{2}\left(\Delta_{J_{2}}\right)=\Delta_{J_{3}}$, with respect to $G=\bigsqcup_{j} a_{j}^{-1} P_{J_{2}}^{w_{1}}$ $=\bigsqcup_{k} P_{J_{3}}^{w_{2} w_{1}} c_{k}$. Consider also the sandwich matrix $\hat{S}=\left(\hat{s}_{k, i}\right)$ of $\left(P_{J_{1}}, L_{J_{1}}, P_{J_{3}}^{w_{2} w_{1}}\right)$ with respect to $G=\bigsqcup_{i} b_{i} P_{J_{1}}=\bigsqcup_{k} P_{J_{3}}^{w_{2} w_{1}} c_{k}$. With this notation we have 
Theorem 4.1. Assume that $l\left(w_{2} w_{1}\right)=l\left(w_{2}\right)+l\left(w_{1}\right)$. Then $\bar{S} S=\hat{S}$.

The proof will be preceded by a sequence of auxiliary lemmas.

Lemma 4.2. Assume that $\alpha>0, w_{2}(\alpha)<0$ and $l\left(w_{2} w_{1}\right)=l\left(w_{2}\right)+l\left(w_{1}\right)$ for some $\alpha \in \Phi, w_{1}, w_{2} \in W$. Then $w_{1}^{-1}(\alpha)>0$.

Proof. We proceed by induction on $l\left(w_{2}\right)$. If $l\left(w_{2}\right)=0$, then the claim is obvious. So suppose $l\left(w_{2}\right)>0$. Then there exist $s_{i}, i \in I$ and $w_{2}^{\prime} \in W$ such that $w_{2}=$ $s_{i} w_{2}^{\prime}$ and $l\left(w_{2}\right)=l\left(w_{2}^{\prime}\right)+1$. Assume first, that $w_{2}^{\prime}(\alpha)>0$. By the hypothesis $s_{i}\left(w_{2}^{\prime}(\alpha)\right)<0$. Hence $w_{2}^{\prime}(\alpha)=\alpha_{i}$. This gives $\alpha=w_{2}^{\prime-1}\left(\alpha_{i}\right)$. So we have to prove that $w_{1}^{-1} w_{2}^{-1}\left(\alpha_{i}\right)>0$. By [1, Prop.2.2.8] this follows from the equality $l\left(w_{1}^{-1} w_{2}^{\prime-1} s_{i}\right)=l\left(w_{1}^{-1} w_{2}^{\prime-1}\right)+1$. It remains to consider the case where $w_{2}^{\prime}(\alpha)<0$. Since $\alpha>0, w_{2}^{\prime}(\alpha)<0$ and $l\left(w_{2}^{\prime} w_{1}\right)=l\left(w_{2}^{\prime}\right)+l\left(w_{1}\right)$, we can apply the induction hypothesis to $w_{2}^{\prime}$ and $w_{1}$ to obtain $w_{1}^{-1}(\alpha)>0$.

Lemma 4.3. Assume, that $w_{1}\left(\Delta_{J_{1}}\right)=\Delta_{J_{2}}, w_{2}\left(\Delta_{J_{2}}\right)=\Delta_{J_{3}}$ and $l\left(w_{2} w_{1}\right)=$ $l\left(w_{2}\right)+l\left(w_{1}\right)$ for some $w_{1}, w_{2} \in W$. Then

(a) $w_{2} U_{J_{2}} w_{1} \subseteq U_{J_{3}} w_{2} w_{1} U_{J_{1}}$,

(b) $w_{2} P_{J_{2}} w_{1} \subseteq P_{J_{3}} w_{2} w_{1} P_{J_{1}}$.

Proof. (a) By [1, Prop.2.5.11] we have $U=U_{w_{0} w_{2}} U_{w_{2}}$. Since $U_{J_{2}}$ is a product of certain root subgroups we have also $U_{J_{2}}=\left(U_{J_{2}} \cap U_{w_{0} w_{2}}\right)\left(U_{J_{2}} \cap U_{w_{2}}\right)$. Hence

$$
\begin{aligned}
w_{2} U_{J_{2}} w_{1} & =w_{2}\left(U_{J_{2}} \cap U_{w_{0} w_{2}}\right)\left(U_{J_{2}} \cap U_{w_{2}}\right) w_{1} \\
& =\left(U_{J_{2}} \cap U_{w_{0} w_{2}}\right)^{w_{2}^{-1}}\left(w_{2} w_{1}\right)\left(U_{J_{2}} \cap U_{w_{2}}\right)^{w_{1}} .
\end{aligned}
$$

First we will prove that $\left(U_{J_{2}} \cap U_{w_{0} w_{2}}\right)^{w_{2}^{-1}} \subseteq U_{J_{3}} . U_{J_{2}} \cap U_{w_{0} w_{2}}$ is a product of $X_{\alpha}$ where $\alpha>0, \alpha \notin \Phi_{J_{2}}$ and $w_{0} w_{2}(\alpha)<0$. This implies that $w_{2}(\alpha)>0$ and $w_{2}(\alpha) \notin w_{2}\left(\Phi_{J_{2}}\right)=\Phi_{J_{3}}$. Hence $X_{\alpha}^{w_{2}^{-1}}=X_{w_{2}(\alpha)} \subseteq U_{J_{3}}$. This proves the desired inclusion. Next, we claim that $\left(U_{J_{2}} \cap U_{w_{2}}\right)^{w_{1}} \subseteq U_{J_{1}} \cdot U_{J_{1}} \cap U_{w_{2}}$ is a product of $X_{\alpha}$ with $\alpha>0, \alpha \notin \Phi_{J_{2}}$ and $w_{2}(\alpha)<0$. By Lemma 4.2 the first and the last condition imply that $w_{1}^{-1}(\alpha)>0$. We have also $w_{1}^{-1}(\alpha) \notin w_{1}^{-1}\left(\Phi_{J_{2}}\right)=\Phi_{J_{1}}$. Hence $X_{\alpha}^{w_{1}} \subseteq U_{J_{1}}$, proving the claim. In view of (3), the two established inclusions imply that (a) holds.

(b) Now, using the first assertion we have

$$
\begin{gathered}
w_{2} P_{J_{2}} w_{1}=w_{2} U_{J_{2}} L_{J_{2}} w_{1}=w_{2} U_{J_{2}} w_{1} L_{J_{2}}^{w_{1}} \subseteq \text { by }(\mathrm{a}) \\
\subseteq U_{J_{3}} w_{2} w_{1} U_{J_{1}} L_{J_{1}} \subseteq P_{J_{3}} w_{2} w_{1} P_{J_{1}} .
\end{gathered}
$$

Lemma 4.4. Assume that $w_{1}\left(\Delta_{J_{1}}\right)=\Delta_{J_{2}}, w_{2}\left(\Delta_{J_{2}}\right)=\Delta_{J_{3}}$ and $l\left(w_{2} w_{1}\right)=l\left(w_{2}\right)+$ $l\left(w_{1}\right)$ for some $w_{1}, w_{2} \in W$. Moreover, if $w_{2} u_{2} w_{1}=u_{3} w_{2} w_{1} u_{1} l$ for some $u_{i} \in$ $U_{J_{i}}, l \in L_{J_{1}}$, then $l=1$.

Proof. By Lemma 4.3(a) there exist $u_{3}^{\prime} \in U_{J_{3}}$ and $u_{1}^{\prime} \in U_{J_{1}}$ such that $w_{2} u_{2} w_{1}=$ $u_{3}^{\prime} w_{2} w_{1} u_{1}^{\prime}$. Hence by the hypothesis $u_{3}^{\prime} w_{2} w_{1} u_{1}^{\prime}=u_{3} w_{2} w_{1} u_{1} l$. This gives $u_{1} l u_{1}^{\prime-1}=$ $\left(w_{2} w_{1}\right)^{-1} u_{3}^{-1} u_{3}^{\prime}\left(w_{2} w_{1}\right)$. So we have $u_{1} l u_{1}^{\prime-1} \in P_{J_{1}} \cap U_{J_{3}}^{w_{2} w_{1}}=U_{J_{1}} \cap U_{J_{3}}^{w_{2} w_{1}}$, the equality following from [1, Prop.2.8.6 and Cor.2.8.8] since $\left(w_{2} w_{1}\right)^{-1}\left(\Delta_{J_{3}}\right)=\Delta_{J_{1}}$. Thus $l \in U_{J_{1}}$. Then $U_{J_{1}} \cap L_{J_{1}}=\{1\}$ implies that $l=1$.

Lemma 4.5. Assume that $w_{1}\left(\Delta_{J_{1}}\right)=\Delta_{J_{2}}, w_{2}\left(\Delta_{J_{2}}\right)=\Delta_{J_{3}}$ and $l\left(w_{2} w_{1}\right)=l\left(w_{2}\right)+$ $l\left(w_{1}\right)$ for some $w_{1}, w_{2} \in W$. Then $U_{J_{3}} \cap U_{J_{1}}^{\left(w_{2} w_{1}\right)^{-1}} \subseteq U_{J_{2}}^{w_{2}^{-1}}$. 
Proof. The assertion is equivalent to

$$
U \cap U^{\left(w_{0}\right)_{J_{3}}} \cap\left(U \cap U^{\left(w_{0}\right)_{J_{1}}}\right)^{\left(w_{2} w_{1}\right)^{-1}} \subseteq\left(U \cap U^{\left(w_{0}\right)_{J_{2}}}\right)^{w_{2}^{-1}}
$$

Hence it is sufficient to show that

(i) $U \cap U^{\left(w_{2} w_{1}\right)^{-1}} \subseteq U^{w_{2}^{-1}}$,

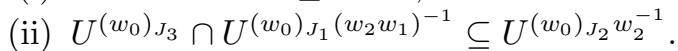

To prove (i) first note that $U \cap U^{\left(w_{2} w_{1}\right)^{-1}}=U_{w_{0}\left(w_{2} w_{1}\right)^{-1}}$ is a product of $X_{\alpha}$ where $\alpha>0, w_{0}\left(w_{2} w_{1}\right)^{-1}(\alpha)<0$. Hence $\left(w_{2} w_{1}\right)^{-1}(\alpha)>0$. If $w_{2}^{-1}(\alpha)<0$ then by Lemma 4.2 (where $\alpha$ stands for $\left.w_{2}^{-1}(-\alpha)\right) w_{1}^{-1} w_{2}^{-1}(\alpha)<0$. This contradiction shows that $w_{2}^{-1}(\alpha)>0$. Therefore $X_{\alpha} \subseteq U^{w_{2}^{-1}}$, which proves the first inclusion.

Next we transform the second inclusion to the form

$$
U^{\left(w_{0}\right)_{J_{3}}} \cap U^{\left(w_{2} w_{1}\right)^{-1}\left(w_{2} w_{1}\right)\left(w_{0}\right)_{J_{1}}\left(w_{2} w_{1}\right)^{-1}} \subseteq U^{w_{2}^{-1} w_{2}\left(w_{0}\right)_{J_{2}} w_{2}^{-1}} .
$$

This is equvalent to

$$
U^{\left(w_{0}\right)_{J_{3}}} \cap U^{\left(w_{2} w_{1}\right)^{-1}\left(w_{0}\right)_{J_{3}}} \subseteq U^{w_{2}^{-1}\left(w_{0}\right)_{J_{3}}} .
$$

Conjugating by $\left(w_{0}\right)_{J_{3}}$ we get (i). So the lemma follows.

Lemma 4.6. Assume that $w_{1}\left(\Delta_{J_{1}}\right)=\Delta_{J_{2}}, w_{2}\left(\Delta_{J_{2}}\right)=\Delta_{J_{3}}$ and $l\left(w_{2} w_{1}\right)=l\left(w_{2}\right)+$ $l\left(w_{1}\right)$ for some $w_{1}, w_{2} \in W$. If moreover $w_{2} u_{2} w_{1}=u_{3} w_{2} w_{1} u_{1}$ for some $u_{i} \in U_{J_{i}}$ then $u_{3} \in U_{J_{2}}^{w_{2}^{-1}}$.

Proof. By the proof of Lemma 4.3(a) there exist $u_{1}^{\prime} \in U_{J_{1}}, u_{3}^{\prime} \in U_{J_{3}} \cap U_{J_{2}}^{w_{2}^{-1}}$ such that $w_{2} u_{1} w_{1}=u_{3}^{\prime} w_{2} w_{1} u_{1}^{\prime}$. Hence by the hypothesis $u_{3}\left(w_{2} w_{1}\right) u_{1}=u_{3}^{\prime}\left(w_{2} w_{1}\right) u_{1}^{\prime}$. This gives $u_{3}^{\prime-1} u_{3}=\left(w_{2} w_{1}\right) u_{1}^{\prime} u_{1}^{-1}\left(w_{2} w_{1}\right)^{-1}$. Thus by Lemma $4.5 u_{3}^{\prime-1} u_{3} \in U_{J_{3}} \cap$ $U_{J_{1}}^{\left(w_{2} w_{1}\right)^{-1}} \subseteq U_{J_{2}}^{w_{2}^{-1}}$. Since $u_{3}^{\prime} \in U_{J_{2}}^{w_{2}^{-1}}$ we have $u_{3} \in U_{J_{2}}^{w_{2}^{-1}}$.

Proof of Theorem 4.1. We have to show that $\sum_{j} \bar{s}_{k, j} s_{j, i}=\hat{s}_{k, i}$ in $K\left[L_{J_{1}}\right]$ for any $k, i$. First, assume that there exists $j_{0}$ with $\bar{s}_{k, j_{0}} \neq 0$ and $s_{j_{0} . i} \neq 0$. In this case $c_{k} a_{j_{0}}^{-1} \in P_{J_{3}}^{w_{2} w_{1}} P_{J_{2}}^{w_{1}}$ and $a_{j_{0}} b_{i} \in P_{J_{2}}^{w_{1}} P_{J_{1}}$. Hence $c_{k} a_{j_{0}}^{-1}=u_{3}^{w_{2} w_{1}} \bar{u}_{2}^{w_{1}} \hat{s}_{k, j_{0}}$ and $a_{j_{0}} b_{i}=s_{j_{0}, i} u_{2}^{w_{1}} u_{1}$ for some $u_{i} \in U_{J_{i}}, \bar{u}_{2} \in U_{J_{2}}$. It follows that

$$
c_{k} b_{i}=c_{k} a_{j_{0}}^{-1} a_{j_{0}} b_{i}=w_{1}^{-1} w_{2}^{-1} u_{3} w_{2} \bar{u}_{2} w_{1} \bar{s}_{k, j_{0}} s_{j_{0}, i} w_{1}^{-1} u_{2} w_{1} u_{1} .
$$

On the other hand

$$
\begin{gathered}
c_{k} b_{i} \in P_{J_{3}}^{w_{2} w_{1}} P_{J_{2}}^{w_{1}} P_{J_{1}}=w_{1}^{-1} w_{2}^{-1} P_{J_{3}}\left(w_{2} P_{J_{2}} w_{1}\right) P_{J_{1}} \subseteq \\
\subseteq w_{1}^{-1} w_{2}^{-1} P_{J_{3}}\left(P_{J_{3}} w_{2} w_{1} P_{J_{1}}\right) P_{J_{1}}=P_{J_{3}}^{w_{2} w_{1}} P_{J_{1}}
\end{gathered}
$$

by Lemma $4.3(\mathrm{~b})$. Thus $c_{k} b_{i}=w_{1}^{-1} w_{2}^{-1} \hat{u}_{3} w_{2} w_{1} \hat{u}_{1} \hat{s}_{k, i}$ for some $\hat{u}_{1} \in U_{J_{1}}, \hat{u}_{3} \in U_{J_{3}}$, which are independent of $j$. Comparing the obtained expressions for $c_{k} b_{i}$ we come to

$$
u_{3} w_{2} \bar{u}_{2}\left(\bar{s}_{k, j_{0}} s_{j_{0}, i}\right)^{w_{1}^{-1}} u_{2} w_{1} u_{1}=\hat{u}_{3} w_{2} w_{1} \hat{u}_{1} \hat{s}_{k, i} .
$$

Hence $u_{3} w_{2}\left(\bar{u}_{2} u_{2}^{*}\right) w_{1}\left(\bar{s}_{k, j_{0}} s_{j_{0}, i}\right) u_{1}=\hat{u}_{3} w_{2} w_{1} \hat{u}_{1} \hat{s}_{k, i}$ where $u_{2}^{*}=u_{2}^{\left(w_{1} \bar{s}_{k, j_{0}} s_{j_{0}, i} w_{1}^{-1}\right)^{-1}} \in$ $U_{J_{2}}$. Therefore

$$
w_{2}\left(\bar{u}_{2} u_{2}^{*}\right) w_{1}=\left(u_{3}^{-1} \hat{u}_{3}\right)\left(w_{2} w_{1}\right)\left(\hat{u}_{1} \hat{s}_{k, i} u_{1}^{-1} \hat{s}_{k, i}^{-1}\right)\left[\hat{s}_{k, i}\left(\bar{s}_{k, j_{0}} s_{j_{0}, i}\right)^{-1}\right] .
$$

Lemma 4.4 implies that $\hat{s}_{k, i}\left(\bar{s}_{k, j_{0}} s_{j_{0}, i}\right)^{-1}=1$. Thus $\bar{s}_{k, j_{0}} s_{j_{0}, i}=\hat{s}_{k, i}$. For any $j$ such that $\bar{s}_{k, j} \neq 0$ and $s_{j, i} \neq 0$ we have

$$
w_{2}\left(\bar{u}_{2} u_{2}^{*}\right) w_{1}=\left(u_{3}^{-1} \hat{u}_{3}\right)\left(w_{2} w_{1}\right)\left(\hat{u}_{1} \bar{s}_{k, i} u_{1}^{-1} \bar{s}_{k, i}^{-1}\right) .
$$


Lemma 4.6 implies $u_{3}^{-1} \hat{u}_{3} \in U_{J_{2}}^{w_{2}^{-1}}$. Hence $u_{3}^{-1} \hat{u}_{3}=w_{2} \tilde{u}_{2}^{-1} w_{2}^{-1}$ for some $\tilde{u}_{2} \in U_{J_{2}}$. This gives $u_{3}=\hat{u}_{3} \tilde{u}_{2}^{w_{2}^{-1}}$. So we have

$$
\begin{gathered}
c_{k} a_{j}^{-1}=w_{1}^{-1} w_{2}^{-1} u_{3} w_{2} \bar{u}_{2} w_{1} \bar{s}_{k, j}=w_{1}^{-1} w_{2}^{-1} \hat{u}_{3} w_{2} \tilde{u}_{2} w_{2}^{-1} w_{2} \bar{u}_{2} w_{1} \bar{s}_{k, j} \\
=\hat{u}_{3}^{w_{2} w_{1}}\left(\tilde{u}_{2} \bar{u}_{2}\right)^{w_{1}} \bar{s}_{k, j} \in \hat{u}_{3}^{w_{2} w_{1}} P_{J_{2}}^{w_{1}} .
\end{gathered}
$$

Hence $a_{j} \in P_{J_{2}}^{w_{1}}\left(\hat{u}_{3}^{-1}\right)^{w_{2} w_{1}} c_{k}$. Since $\hat{u}_{3}$ is independent of $j$, the index $j$ is determined uniquely. Hence $\sum_{j} \bar{s}_{k, j} s_{j, i}=\bar{s}_{k, j_{0}} s_{j_{0}, i}=\hat{s}_{k, i}$.

It remains to consider the case where, for every $j, \bar{s}_{k, j}=0$ or $s_{j, i}=0$. We have to prove that $\hat{s}_{k, i}=0$. If $\hat{s}_{k, i} \neq 0$ then there exist $\hat{u}_{1} \in U_{J_{1}}$ and $\hat{u}_{3} \in U_{J_{3}}$ such that $c_{k} b_{i}=w_{1}^{-1} w_{2}^{-1} \hat{u}_{3} w_{2} w_{1} \hat{u}_{1} \hat{s}_{k, i}$. Choose $j$ with $a_{j} \in P_{J_{2}}^{w_{1}}\left(\hat{u}_{3}^{-1}\right)^{w_{2} w_{1}} c_{k}$. Then $c_{k} a_{j}^{-1} \in P_{J_{3}}^{w_{2} w_{1}} P_{J_{2}}^{w_{1}}$, hence $\bar{s}_{k, j} \neq 0$. Moreover

$$
\begin{gathered}
a_{j} b_{i}=\left(a_{j} c_{k}^{-1}\right)\left(c_{k} b_{i}\right)=\left(a_{j} c_{k}^{-1}\right)\left(\hat{u}_{3}^{w_{2} w_{1}}\right) \hat{u}_{1} \hat{s}_{k, i} \\
\in P_{J_{2}}^{w_{1}}\left(\hat{u}_{3}^{-1}\right)^{w_{2} w_{1}} \hat{u}_{3}^{w_{2} w_{1}} \hat{u}_{1} \hat{s}_{k, i} \subseteq P_{J_{2}}^{w_{1}} P_{J_{1}} .
\end{gathered}
$$

This implies that $s_{j, i} \neq 0$. This contradiction completes the proof.

Our last aim is to obtain a useful decomposition of the sandwich matrix. Let $J \subseteq I$ and $\alpha \in \Delta \backslash \Delta_{J}$. We then define $w_{\alpha, J} \in W$ by $w_{\alpha, J}=\left(w_{0}\right)_{J \cup\{\alpha\}}\left(w_{0}\right)_{J}$. Recall that $\left(w_{0}\right)_{J \cup\{\alpha\}}$ and $\left(w_{0}\right)_{J}$ are the elements of maximal length in the Coxeter groups with simple root systems $\Delta_{J} \cup\{\alpha\}$ and $\Delta_{J}$ respectively. Let $J_{1}, J^{\prime} \subseteq I, w \in W$ and $w\left(\Delta_{J_{1}}\right)=\Delta_{J^{\prime}}$. Then by $[1$, Prop.10.7.2] $w$ can be expressed in the form $w=w_{\alpha_{k}, J_{k}} \ldots w_{\alpha_{1}, J_{1}}$ where $l(w)=l\left(w_{\alpha_{k}, J_{k}}\right)+\ldots+l\left(w_{\alpha_{1}, J_{1}}\right), \alpha_{1}, \ldots, \alpha_{k} \in \Delta$ and $J_{1}, \ldots, J_{k} \subseteq I$ are such that $w_{\alpha_{i}, J_{i}}\left(\Delta_{J_{i}}\right)=\Delta_{J_{i+1}}$ and $J_{k+1}=J^{\prime}$. Hence by Theorem 4.1 the sandwich matrix of $\left(P_{J_{1}}, L_{J_{1}}, P_{J^{\prime}}^{w}\right)=\left(P_{J_{1}}, L_{J_{1}}, P_{J^{\prime}}^{w_{\alpha_{k}}, J_{k} \ldots w_{\alpha_{1}}, J_{1}}\right)$ may be expressed as a product of matrices which are conjugate by some elements of $W$ to sandwich matrices of type $\left(P_{J_{1}}, L_{J_{1}}, P_{J_{2}}^{w_{\alpha}, J_{1}}\right)$ where $w_{\alpha, J_{1}}\left(\Delta_{J_{1}}\right)=\Delta_{J_{2}}, J_{1}, J_{2} \subseteq I$. In particular, the problem of finding the inverse of the sandwich matrix is reduced to this case. We will investigate the sandwich matrix of the latter type. We start by choosing convenient coset representatives for parabolic subgroups.

Lemma 4.7. Consider coset decompositions $L_{J_{1} \cup\{\alpha\}}=\bigsqcup_{i} b_{i}\left(P_{J_{1}} \cap L_{J_{1} \cup\{\alpha\}}\right)=$ $\bigsqcup_{j}\left(P_{J_{2}}^{w_{\alpha} J_{1}} \cap L_{J_{1} \cup\{\alpha\}}\right) a_{j}$ and $G=\bigsqcup_{k} c_{k} P_{J_{1} \cup\{\alpha\}}$. Then $G$ is decomposed as follows: $G=\bigsqcup_{k, i} c_{k} b_{i} P_{J_{1}}=\bigsqcup_{j, l} P_{J_{2}}^{w_{\alpha} J_{1}} a_{j} c_{l}^{-1}$

Proof. First we show that the cosets $c_{k} b_{i} P_{J_{1}}$ are disjoint (similarly, one shows that $P_{J_{2}}^{w_{\alpha} J_{1}} a_{j} c_{l}^{-1}$ are disjoint). Assume that $c_{k_{1}} b_{i_{1}} P_{J_{1}}=c_{k_{2}} b_{i_{2}} P_{J_{1}}$. Then $b_{i_{2}}^{-1} c_{k_{2}}^{-1} c_{k_{1}} b_{i_{1}} \in$ $P_{J_{1}}$. Hence $c_{k_{2}}^{-1} c_{k_{1}} \in b_{i_{2}} P_{J_{1}} b_{i_{1}}^{-1} \subseteq P_{J_{1} \cup\{\alpha\}}$. By the hypothesis we have $k_{1}=k_{2}$. So $b_{i_{2}}^{-1} b_{i_{1}} \in P_{J_{1}} \cap L_{J_{1} \cup\{\alpha\}}$. This gives $i_{1}=i_{2}$. Next, we prove that $G=\bigcup_{k, i} c_{k} b_{i} P_{J_{1}}$ and $G=\bigcup_{j, l} P_{J_{2}}^{w_{\alpha}, J_{1}} a_{j} c_{l}^{-1}$. If $g \in G$ then $g \in c_{k} P_{J_{1} \cup\{\alpha\}}$ for some $k$. Hence $g=c_{k} u l$ for some $u \in U_{J_{1} \cup\{\alpha\}}, l \in L_{J_{1} \cup\{\alpha\}}$. Similarly $l \in b_{i}\left(P_{J_{1}} \cap L_{J_{1} \cup\{\alpha\}}\right)$ for some $i$, so $l=b_{i} p$ where $p \in P_{J_{1}} \cap L_{J_{1} \cup\{\alpha\}}$. Then we have $g P_{J_{1}}=c_{k} u l P_{J_{1}}=c_{k} u b_{i} p P_{J_{1}}=$ $c_{k} b_{i}\left(b_{i}^{-1} u b_{i}\right) P_{J_{1}}=c_{k} b_{i} P_{J_{1}}$ since $b_{i}^{-1} u b_{i} \in U_{J_{1} \cup\{\alpha\}}$. Now, consider the second coset decomposition. Let $g \in G$. Then $g \in P_{J_{1} \cup\{\alpha\}} c_{k}^{-1}$ for some $k$. Hence $g=u l c_{k}^{-1}$

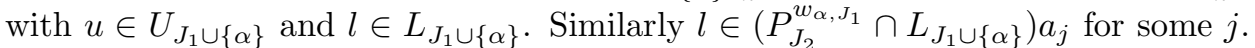
Thus $l=p a_{j}$ where $p \in P_{J_{2}}^{w_{\alpha} J_{1}} \cap L_{J_{1} \cup\{\alpha\}}$. Then we have $P_{J_{2}}^{w_{\alpha} J_{1}} g=P_{J_{2}}^{w_{\alpha} J_{1}} u l c_{k}^{-1}=$

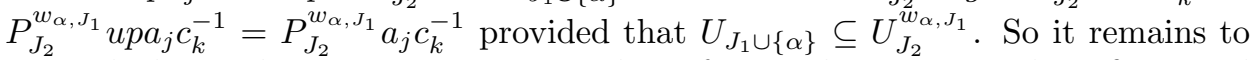
prove the last inclusion. $U_{J_{1} \cup\{\alpha\}}$ is a product of root subgroups $X_{\beta}$ where $\beta>0$ and 
$\beta \notin \Phi_{J_{1} \cup\{\alpha\}}$. Hence $\left(w_{0}\right)_{J_{1}}(\beta)>0$. This implies that $\left(w_{0}\right)_{J_{1} \cup\{\alpha\}}\left(\left(w_{0}\right)_{J_{1}}(\beta)\right)>0$ since $\left(w_{0}\right)_{J_{1}}(\beta) \notin \Phi_{J_{1} \cup\{\alpha\}}$. Therefore $w_{\alpha, J_{1}}(\beta)>0$. Moreover $w_{\alpha, J_{1}}(\beta) \notin \Phi_{J_{2}}$ since $w_{\alpha, J_{1}}\left(\Delta_{J_{1}}\right)=\Delta_{J_{2}}$. So $X_{\beta}^{w_{\alpha, J_{1}}^{-1}} \subseteq U_{J_{2}}$. This gives $X_{\beta} \subseteq U_{J_{2}}^{w_{\alpha, J_{1}}}$. Thus the lemma follows.

Let $D=\left(d_{(j, l),(k, i)}\right)$ be the sandwich matrix of $\left(P_{J_{1}}, L_{J_{1}}, P_{J_{2}, J_{1}}^{w_{\alpha}}\right)$ with respect to the following coset decompositions $G=\bigsqcup_{k, i} c_{k} b_{i} P_{J_{1}}=\bigsqcup_{j, l} P_{J_{2}}^{w_{\alpha, J_{1}}} a_{j} c_{l}^{-1}$. Let $E=$ $\left(e_{j, i}\right)$ be the sandwich matrix of the monoid defined by $\left(P_{J_{1}} \cap L_{J_{1} \cup\{\alpha\}}, L_{J_{1}}, P_{J_{2}}^{w_{\alpha}, J_{1}} \cap\right.$ $L_{J_{1} \cup\{\alpha\}}$ ) (with the group of units $G=L_{J_{1} \cup\{\alpha\}}$ ) with respect to the coset decompositions $L_{J_{1} \cup\{\alpha\}}=\bigsqcup_{i} b_{i}\left(P_{J_{1}} \cap L_{J_{1} \cup\{\alpha\}}\right)=\bigsqcup_{j}\left(P_{J_{2}}^{w_{\alpha}, J_{1}} \cap L_{J_{1} \cup\{\alpha\}}\right) a_{j}$. With this notation the structure of $D$ can be described as follows.

Proposition 4.8. We have

(a) $d_{(j, l),(k, i)}=0$ for $k \neq l$,

(b) $d_{(j, k),(k, i)}=e_{j, i}$.

That is, $D$ has a block diagonal form with the diagonal blocks equal to $E$.

Proof. Assume that $d_{(j, l),(k, i)} \neq 0$. Then $a_{j} c_{l}^{-1} c_{k} b_{i} \in P_{J_{2}}^{w_{\alpha} J_{1}} P_{J_{1}}$. Hence $c_{l}^{-1} c_{k} \in$ $a_{j}^{-1} P_{J_{2}}^{w_{\alpha} J_{1}} P_{J_{1}} b_{i}^{-1} \subseteq P_{J_{1} \cup\{\alpha\}}$ since $P_{J_{2}, J_{1}}^{w_{1}} P_{J_{1}} \subseteq P_{J_{1} \cup\{\alpha\}}$. This implies that $k=l$, so (a) is proved. This gives $a_{j} b_{i} \in P_{J_{2}}^{w_{\alpha, J_{1}}} P_{J_{1}}$. Hence $a_{j} b_{i}=u_{2}^{w_{\alpha, J_{1}}} u_{1} d_{(j, l),(k, i)}$ for some $u_{i} \in U_{J_{i}}$. Since $u_{2}^{w_{\alpha, J_{1}}}, u_{1} \in P_{J_{1} \cup\{\alpha\}}$ then $u_{2}^{w_{\alpha, J_{1}}}=\bar{u}_{2} l, u_{1}=\bar{u}_{1} \bar{l}$ for some $\bar{u}_{i} \in U_{J_{1} \cup\{\alpha\}}$ and $l, \bar{l} \in L_{J_{1} \cup\{\alpha\}}$. By the last part of the proof of Lemma 4.7 we have $U_{J_{1} \cup\{\alpha\}} \subseteq U_{J_{2}}^{w_{\alpha, J_{1}}}$. Hence $l \in U_{J_{2}}^{w_{\alpha, J_{1}}} \cap L_{J_{1} \cup\{\alpha\}}$. Similarly $\bar{l} \in U_{J_{1}} \cap L_{J_{1} \cup\{\alpha\}}$ since $U_{J_{1} \cup\{\alpha\}} \subseteq U_{J_{1}}$. So we have $a_{j} b_{i}=\bar{u}_{2} l \bar{u}_{1} \bar{l} d_{(j, l),(k, i)}=\bar{u}_{2}\left(l \bar{u}_{1} l^{-1}\right)\left(l \bar{l} d_{(j, l),(k, i)}\right)$. Since $a_{j} b_{i} \in L_{J_{1} \cup\{\alpha\}}$ then $\bar{u}_{2}\left(l \bar{u}_{1} l^{-1}\right)=1$. Hence

$$
a_{j} b_{i}=l \bar{l} d_{(j, l),(k, i)} \in\left(P_{J_{2}}^{w_{\alpha, J_{1}}} \cap L_{J_{1} \cup\{\alpha\}}\right)\left(P_{J_{1}} \cap L_{J_{1} \cup\{\alpha\}}\right)
$$

with $l \in U_{J_{2}}^{w_{\alpha, J_{1}}} \cap L_{J_{1} \cup\{\alpha\}}, \bar{l} \in U_{J_{1}} \cap L_{J_{1} \cup\{\alpha\}}$. This proves that $d_{(j, l),(k, i)}=e_{j, i}$ $(k=l)$ in the case where $d_{(j, k),(k, i)} \neq 0$. If $d_{(j, k),(k, i)}=0$ then $e_{j, i}=0$ by the definition, so we have also $d_{(j, k),(k, i)}=e_{j, i}$. This completes the proof of (b).

Since $P_{J_{1}} \cap L_{J_{1} \cup\{\alpha\}}, P_{J_{2}}^{w_{\alpha, J_{1}}} \cap L_{J_{1} \cup\{\alpha\}}$ are maximal parabolic subgroups of $L_{J_{1} \cup\{\alpha\}}$ with common Levi factor $L_{J_{1}}$, they are also opposite. Therefore, by the paragraph preceding Lemma 4.7 together with this lemma and Proposition 4.8, one is reduced to considering monoids $M=M\left(G, P, P^{-}, L\right)$ where $P$ and $P^{-}$are opposite and maximal parabolic subgroups of $G$.

\section{REFERENCES}

[1] Carter,R.W., Finite Groups of Lie Type : Conjugacy Classes and Complex Characters, Wiley, 1985. MR 87d:20060

[2] Clifford,A.H. and Preston,G.B., The Algebraic Theory of Semigroups, Math. Surveys, no.7, Amer. Math. Soc., Providence, R.I., 1961. MR 24:A2627

[3] Curtis,C.W. and Reiner,I., Methods of Representation Theory, Volumes 1,2, Wiley, New York, 1987. MR 88f:20002

[4] Howlett,R.B. and Lehrer,G.L., Induced cuspidal representations and generalised Hecke rings, Invent. Math. 58 (1980), 37-64. MR 81j:20017

[5] Kovacs,L.G., Semigroup algebras of the full matrix semigroups over a finite field, Proc. Amer. Math. Soc. 116 (1992), 911-917. MR 93b:16051

[6] Okninski,J. and Putcha,M.S., Complex representations of matrix semigroups, Trans. Amer. Math. Soc. 323 (1991), 563-581. MR 91e:20047 
[7] Putcha,M.S., Monoids on groups with BN-pair, J. Algebra 120 (1989), 139-169. MR 89k:20091

[8] Putcha,M.S., Classification of monoids of Lie type, J. Algebra 163 (1994), 636-662. MR 95b:20089

[9] Putcha,M.S., Sandwich matrices, Solomon algebras and Kazhdan-Lusztig polynomials, Trans. Amer. Math. Soc. 340 (1993), 415-428. MR 94a:20112

[10] Putcha,M.S., Monoid Hecke algebras, preprint.

[11] Putcha,M.S., Big cell algebras I. Steinberg representation, preprint.

[12] Solomon,L., The Bruhat decomposition, Tits system and Iwahori ring for the monoid of matrices over a finite field, Geom. Dedicata 36 (1990), 15-39. MR 92e:20035

Institute of Mathematics, University of Warsaw, Banacha 2,02-097 Warsaw, Poland E-mail address: asalwa@mimuw.edu.pl 